TRANSACTIONS OF THE

AMERICAN MATHEMATICAL SOCIETY

Volume 352, Number 5, Pages 2179-2204

S 0002-9947(00)02611-8

Article electronically published on February 16, 2000

\title{
TOPOLOGICAL HOCHSCHILD HOMOLOGY OF NUMBER RINGS
}

\author{
AYELET LINDENSTRAUSS AND IB MADSEN
}

\begin{abstract}
We calculate an explicit formula for the topological Hochschild homology of a discrete valuation ring of characteristic zero with finite residue field. From this we deduce the topological Hochschild homology of global number rings.
\end{abstract}

\section{INTRODUCTION}

Let $K$ be a finite extension of the field of rational numbers, and $A$ its ring of integers. This paper calculates the topological Hochschild homology groups $\mathrm{THH}_{*}(A)$ in terms of the different ideal of $K$ over $\mathbf{Q}$. More precisely, let $\mathcal{D}_{A}{ }^{-1}$ be the inverse different, that is, the maximal sub- $A$-module of $K$ whose trace is integral.

Theorem 1.1. The non-zero topological Hochschild homology groups of $A$ are

$$
\mathrm{THH}_{0}(A)=A, \quad \mathrm{THH}_{2 n-1}(A)=\mathcal{D}_{A}^{-1} / n A .
$$

There are many good descriptions of the different; $c f$. [S], chapter III. For example, it is the annihilator ideal of the Kähler differentials $\Omega_{A / \mathbf{Z}}$, and $\mathcal{D}_{A}{ }^{-1} / A$ is the first Hochschild homology group of $A$.

Let $R$ be any ring, and let $M$ be an $R$-bimodule. Consider the simplicial abelian group HH. $(R, M)$ with $n$-simplices $M \otimes R^{\otimes n}$, and the standard Hochschild-type face and degeneracy operators. The homology groups of the associated chain complex, or equivalently, the homotopy groups of its topological realization $\operatorname{HH}(R, M)$, are the Hochschild homology groups. In particular, $\mathrm{HH}_{0}(R, M)=M /[R, M]$.

The topological Hochschild homology groups were defined by M. Bökstedt in $[\mathrm{B}]$, but are closely related to results from $[\mathrm{Br}$. They are the homotopy groups of a simplicial spectrum THH. $(R, M)$, which, roughly speaking, has $n$-simplices $\mathrm{H} M \wedge$ $\mathrm{H} R \wedge \cdots \wedge \mathrm{H} R$ where $\mathrm{H} R$ denotes the Eilenberg-Mac Lane spectrum associated with $R$.

The special case $M=R$, where we write $\operatorname{THH}(R)$ instead of $\operatorname{THH}(R, M)$, is of particular importance. Connes' theory of cyclic objects implies that $\mathrm{THH}(R)$ has a continuous circle action, and there is a further construction $\mathrm{TC}(R)$, the topological cyclic homology spectrum, whose homotopy groups are intimately related to Quillen's higher K-groups, $c f .[\mathrm{HM},[\mathbf{M}$. It is hoped that the above theorem might eventually lead to a calculation of $\mathrm{TC}_{*}(A)$ for the rings of Theorem 1.1 .

Received by the editors December 18, 1997.

2000 Mathematics Subject Classification. Primary 19D55; Secondary 13D03, 19D50, 55Q52. 
There are a number of alternative descriptions of $\operatorname{THH}(R, M)$, interesting in their own right, which will be important in the proof of the theorem.

Let $\mathcal{F}_{R}$ denote the category of functors from $P_{R}$ to $R$-mod, where $P_{R}$ denotes the category of finitely generated right $R$-modules. Each $R$-bimodule $M$ gives an element of $\mathcal{F}_{R}$, namely the functor $T_{M}(P)=P \otimes_{R} M$, and this inclusion has a left-adjoint $L: \mathcal{F}_{R} \rightarrow R$-bimod. Concretely, $L T$ is the cokernel of

$$
T d_{0}-T d_{1}+T d_{2}: T(R \times R) \rightarrow T(R),
$$

where $d_{0}\left(r_{0}, r_{1}\right)=r_{1}, d_{1}\left(r_{0}, r_{1}\right)=r_{0} r_{1}$, and $d_{2}\left(r_{0}, r_{1}\right)=r_{0}(T(R)$ and hence the cokernel is a right $R$-module, and the left action on the cokernel is induced from $T(s \mapsto r s))$.

The functor $\mathrm{HH}_{0}(R, L T)$ on $\mathcal{F}_{R}$ is right exact. It is a theorem of Pirashvili and Waldhausen [PW] that the associated left derived functors in $\mathcal{F}_{R}$ are the topological Hochschild homology groups,

$$
L_{n} \operatorname{HH}_{0}\left(R, L T_{M}\right)=\mathrm{THH}_{n}(R, M)
$$

with $T_{M} \in \mathcal{F}_{R}$ as above.

This generalizes the well-known result that the $\mathrm{HH}_{n}(R, M)$ are the left derived functors of $\mathrm{HH}_{0}(R, M)$, where $R$ is a $k$-algebra, and $k$ a field.

For any small linear category $\mathcal{C}$ and bi-functor

$$
\mathcal{M}: \mathcal{C}^{\text {op }} \times \mathcal{C} \rightarrow \text { abelian groups }
$$

one has homology groups $H_{*}(\mathcal{C}, \mathcal{M})$ (see e.g. [DM1]). They may be defined in analogy with $\mathrm{HH}_{*}(R, M)$ as follows. Given a pointed set $X$, we write

$$
\mathbf{Z}(X)=\mathbf{Z}[X] / \mathbf{Z} \cdot *,
$$

where $\mathbf{Z}[X]$ is the abelian group with basis $X$. Consider the simplicial abelian group $V .(\mathbf{Z C}, \mathcal{M})$ with $n$-simplices

$$
V_{n}(\mathbf{Z C}, \mathcal{M})=\bigoplus \mathcal{M}\left(c_{0}, c_{r}\right) \otimes \mathbf{Z}\left(\mathcal{C}\left(c_{1}, c_{0}\right)\right) \otimes \cdots \otimes \mathbf{Z}\left(\mathcal{C}\left(c_{r}, c_{r-1}\right)\right),
$$

where the direct sum is over objects $c_{0}, \ldots, c_{r}$ in $\mathcal{C}$ and where $\mathcal{C}(c, d)$ is the set of morphisms, with 0 as basepoint. The standard Hochschild-type face and degeneracy operators define the simplicial structure; on generators of the form $m \otimes f_{1} \otimes \cdots \otimes f_{r}$,

$$
\begin{aligned}
d_{0}\left(m \otimes f_{1} \otimes \cdots \otimes f_{r}\right) & =\left(f_{1}^{\mathrm{op}}\right)_{*}(m) \otimes f_{2} \otimes \cdots \otimes f_{r}, \\
d_{i}\left(m \otimes f_{1} \otimes \cdots \otimes f_{r}\right) & =m \otimes f_{1} \otimes \cdots \otimes f_{i+1} \circ f_{i} \otimes \cdots \otimes f_{r}, \\
d_{r}\left(m \otimes f_{1} \otimes \cdots \otimes f_{r}\right) & =\left(f_{r}\right)_{*}(m) \otimes f_{1} \otimes \cdots \otimes f_{r-1},
\end{aligned}
$$

and

$$
H_{*}(\mathcal{C}, \mathcal{M})=\pi_{*} V(\mathbf{Z C}, \mathcal{M}) .
$$

If $\mathcal{C}=R$ is the category with one element and the ring $R$ as its morphisms, then $\mathcal{M}$ is a bimodule $M$ and the definition reduces to the standard cyclic construction of $\mathbf{Z}(R)$ with coefficients in $M$ considered as a $\mathbf{Z}(R)$-bimodule,

$$
V_{k}(\mathbf{Z}(R), M)=M \otimes \mathbf{Z}(R)^{\otimes k} .
$$

Thus the homotopy groups become $\mathrm{HH}_{*}(\mathbf{Z}(R), M)$.

If one takes $\mathcal{C}=P_{R}$, on the other hand, and lets $\mathcal{M}=\operatorname{Hom}_{R}\left(P, Q \otimes_{R} M\right)$, then a theorem of Jibladze and Pirashvili [JP] asserts that

$$
H_{*}\left(P_{R}, \operatorname{Hom}_{R}\left(-,-\otimes_{R} M\right)\right)=L_{n} \operatorname{HH}_{0}\left(R, L T_{M}\right),
$$


which combined with formula (1.1) gives another view of $\mathrm{THH}_{*}(R, M)$.

For $A=\mathbf{Z}$, Theorem 1.1 is a celebrated result of M. Bökstedt, [B1], [FP. For $|K: \mathbf{Q}|=2$ ramified only at 2 , the theorem is proved in [L1] by different methods. If $K$ is a local field which is unramified over $\mathbf{Q}_{p}$, so that $A$ is the ring of Witt vectors of $\mathbf{F}_{p^{s}}$, then the $p$-torsion in the theorem can easily be derived from [BM], where $\mathrm{TC}_{*}(A)$ was also calculated.

The proof we present is based on a spectral sequence due to Morten Brun, which in turn is dual to a spectral sequence of Pirashvili. It is a multiplicative homologytype spectral sequence,

$$
\operatorname{THH}_{*}\left(A / p, \operatorname{Tor}_{*}^{A}(A / p, A / p)\right) \Rightarrow \operatorname{THH}_{*}(A, A / p) .
$$

In section 4 below we completely determine the spectral sequence for the rings of theorem 1.1 The homotopy groups $\operatorname{THH}_{*}(A, M)$, considered as a functor of $M$, send a short exact sequence of bimodules into a long exact sequence, e.g. by formula (1.1), so in particular

$$
\mathrm{THH}_{*}(A, A / p) \cong \pi_{*}(\operatorname{THH}(A) ; \mathbf{Z} / p),
$$

homotopy with coefficients in $\mathbf{Z} / p$. Theorem 1.1 then follows from the knowledge of $\mathrm{THH}_{*}(A, A / p)$ by an evaluation of the Bockstein spectral sequence. This is carried out in section 5 .

In many places below we shall use multisimplicial sets and their realizations, so we now remind the reader that a multisimplicial set, say $X_{. .,}$, gives rise to a simplicial set $\delta X$., its diagonal, and that the topological realization $\mid \delta X$.| is homeomorphic to the twofold realizations

$$
|\delta X .| \cong|k \mapsto| X_{k, \cdot}|| \cong|\ell \mapsto| X_{\cdot, \ell}|| .
$$

Usually we shall drop the $\delta$ from the notation, and freely consider a multisimplicial set as a (multi-) simplicial set by taking the diagonal with respect to (some of) the directions.

Finally, it is a pleasure to acknowledge the help we have had from B. Dundas, and to thank Morten Brun for showing us the spectral sequence on which this paper is based. Not least we thank T. Pirashvili for pointing out that the conjecture (5.4.2) in [M] was indeed correct (at least additively) by an application of his spectral sequence, dual to the above. This was the starting point of the approach in the present paper.

\section{Simplicial Constructions}

Let $C$ be a monad in the category of endofunctors of s.sets, the category of pointed simplicial sets, and let $E$ and $F$ be a right and a left $C$-module. Thus we have maps

$$
\begin{array}{ll}
\mu: C \circ C \rightarrow C, & \iota: \mathrm{id} \rightarrow C, \\
\ell: C \circ F \rightarrow F, & r: E \circ C \rightarrow E,
\end{array}
$$

which satisfy the obvious identities, $c f$. [Mac. We can form the two-sided bar construction $\mathcal{B}$. $(E, C, F)$ with $k$-simplices

$$
\mathcal{B}_{k}(E, C, F)=E \circ C^{(k)} \circ F, \quad C^{(k)}=C \circ \cdots \circ C,
$$

and the standard face and degeneracy operators induced from $r, \mu, \ell$, and $\iota$. One can evaluate $\mathcal{B} .(E, C, F)$ on a pointed simplicial set $X$. to get a bisimplicial set whose 
realization is denoted by $\mathcal{B}$. $(E, C, F)(X)$. If $E=C$ there is an "extra degeneracy operator", and $\ell$ defines an augmentation

$$
\mathcal{B} .(C, C, F) \rightarrow F
$$

that becomes a homotopy equivalence upon evaluating at any simplicial set $X$. Indeed the extra degeneracy operator $s_{-1}: C^{(k)} \circ F \rightarrow C^{(k+1)} \circ F$ is equal to $\iota \circ C^{(k)} \circ F$, and there are maps

$$
\ell \circ d_{0}^{(\cdot)}: \mathcal{B} .(C, C, F) \rightarrow F, \quad s^{(\cdot)} \circ s_{-1}: F \rightarrow \mathcal{B} .(C, C, F),
$$

for which one composition is the identity, and the other composition is homotopic to the identity by the homotopy

$$
h_{k}: \mathcal{B}_{k-1}(C, C, F) \rightarrow \mathcal{B}_{k}(C, C, F)
$$

defined to be $h_{0}=s_{-1}, h_{1}=s_{0} s_{-1} d_{0}, h_{2}=s_{0}^{2} s_{-1} d_{0}^{2}$, etc. (cf. May1, definition 5.1).

Given a ring $R$ and a pair $M, N$ consisting of a right and and a left $R$-module, we get into the situation above by defining functors

$$
R\left(X_{.}\right)=R\left[X_{.}\right] / R \cdot *, M\left(X_{.}\right)=M\left[X_{.}\right] / M \cdot *, N\left(X_{.}\right)=N\left[X_{.}\right] / N \cdot * .
$$

In this case we write $B .(M, R, N)$ for the construction evaluated at $S_{.}^{0}$, the 0 dimensional simplicial sphere consisting of two points. Since the chain complex $B_{*}(R, R, N)$ is the standard $R$-free resolution of $N$, and

$$
B_{*}(M, R, N)=M \otimes_{R} B_{*}(R, R, N),
$$

the homology groups of $B_{*}(M, R, N)$ are the Tor-groups, or alternatively

$$
\pi_{n} B .(M, R, N)=\operatorname{Tor}_{n}^{R}(M, N) .
$$

Next we consider the simplicial abelian group $W .(M, \mathbf{Z}(R), \mathbf{Z}(N))$, the usual (tensor product) two-sided bar construction of $\mathbf{Z}(R)$ with right module $M$ and left module $\mathbf{Z}(N)$. It has $k$-simplices

$$
W_{k}(M, \mathbf{Z}(R), \mathbf{Z}(N))=M \otimes \mathbf{Z}(R) \otimes \cdots \otimes \mathbf{Z}(R) \otimes \mathbf{Z}(N)
$$

with $\mathbf{Z}(R)=\mathbf{Z}[R] / \mathbf{Z} \cdot 0$ as above, and with the usual bar-type face and degeneracy operators induced from

$$
\begin{aligned}
M \otimes \mathbf{Z}(R) & =M(R) \stackrel{\mathrm{ev}}{\longrightarrow} M \\
\mathbf{Z}(R) \otimes \mathbf{Z}(R) & =\mathbf{Z}(R \wedge R) \stackrel{\mathbf{Z}(\text { mult })}{\longrightarrow} \mathbf{Z}(R), \\
\mathbf{Z}(R) \otimes \mathbf{Z}(N) & =\mathbf{Z}(R \wedge N) \stackrel{\mathbf{Z}(\text { mult })}{\longrightarrow} \mathbf{Z}(N) .
\end{aligned}
$$

Thus

$$
\pi_{n} W(M, \mathbf{Z}(R), \mathbf{Z}(N))=\operatorname{Tor}_{n}^{\mathbf{Z}(R)}(M, \mathbf{Z}(N))
$$

We can use the maps

$$
R \wedge N \rightarrow R(N), \quad(r, n) \mapsto r \cdot n,
$$

and the identifications

$$
\mathbf{Z}(R) \otimes \mathbf{Z}(N)=\mathbf{Z}(R \wedge N), \quad M \otimes \mathbf{Z}(R)=M(R)
$$

to define a simplicial map

$$
\text { ev }: W .(M, \mathbf{Z}(R), \mathbf{Z}(N)) \rightarrow B .(M, R, N) .
$$


It induces on homotopy groups the map

$$
\mathrm{ev}_{*}: \operatorname{Tor}_{n}^{\mathbf{Z}(R)}(M, \mathbf{Z}(N)) \rightarrow \operatorname{Tor}_{n}^{R}(M, N)
$$

associated with the evaluations $\mathbf{Z}(R) \rightarrow R, \mathbf{Z}(N) \rightarrow N$.

Let $\pi: R \rightarrow R_{1}$ be a homomorphism of rings. Then the $\ell$-simplices

$$
W_{\ell}\left(R_{1}, \mathbf{Z}(R), \mathbf{Z}\left(R_{1}\right)\right)
$$

form a left $R_{1}$-module and a right $\mathbf{Z}\left(R_{1}\right)$-module and so can be viewed as a $\mathbf{Z}\left(R_{1}\right)$ bimodule. Thus we have a bisimplicial abelian group

$$
V .\left(\mathbf{Z}\left(R_{1}\right), W .\left(R_{1}, \mathbf{Z}(R), \mathbf{Z}\left(R_{1}\right)\right)\right)
$$

with $(k, \ell)$-simplices

$$
W_{\ell}\left(R_{1}, \mathbf{Z}(R), \mathbf{Z}\left(R_{1}\right)\right) \otimes \mathbf{Z}\left(R_{1}\right)^{\otimes k}
$$

with the Hochschild-type face and degeneracy operators in the $k$ direction and bar-type operators in the $\ell$ direction. Now

$$
\begin{aligned}
V_{k}\left(\mathbf{Z}\left(R_{1}\right), W_{\ell}\left(R_{1}, \mathbf{Z}(R), \mathbf{Z}\left(R_{1}\right)\right)\right) & =R_{1} \otimes \mathbf{Z}(R)^{\otimes \ell} \otimes \mathbf{Z}\left(R_{1}\right)^{\otimes(k+1)} \\
& \cong V_{\ell}\left(\mathbf{Z}(R), W_{k}\left(\mathbf{Z}\left(R_{1}\right), \mathbf{Z}\left(R_{1}\right), R_{1}\right)\right),
\end{aligned}
$$

where the isomorphism is by the following rearrangement of tensor factors:

$r_{1} \otimes z_{1} \otimes \cdots \otimes z_{\ell} \otimes w_{0} \otimes w_{1} \otimes \cdots \otimes w_{k} \mapsto w_{0} \otimes w_{1} \otimes \cdots \otimes w_{k} \otimes r_{1} \otimes z_{1} \otimes \cdots \otimes z_{\ell}$.

Since $W .\left(\mathbf{Z}\left(R_{1}\right), \mathbf{Z}\left(R_{1}\right), R_{1}\right)$ is homotopy equivalent to $R_{1}$,

$$
V .\left(\mathbf{Z}\left(R_{1}\right), W .\left(\mathbf{Z}\left(R_{1}\right), \mathbf{Z}(R), R_{1}\right)\right) \simeq V_{.}\left(\mathbf{Z}(R), R_{1}\right) .
$$

The homology groups of $V_{*}\left(\mathbf{Z}(R), R_{1}\right)$ are the Hochschild homology groups $\mathrm{HH}_{*}\left(\mathbf{Z}(R), R_{1}\right)$, so the double complex $V_{*}\left(\mathbf{Z}\left(R_{1}\right), W_{*}\left(R_{1}, \mathbf{Z}(R), \mathbf{Z}\left(R_{1}\right)\right)\right)$ gives rise to a spectral sequence

$$
\mathrm{HH}_{*}\left(\mathbf{Z}\left(R_{1}\right), \operatorname{Tor}_{*}^{\mathbf{Z}(R)}\left(R_{1}, \mathbf{Z}\left(R_{1}\right)\right)\right) \Rightarrow \mathrm{HH}_{*}\left(\mathbf{Z}(R), R_{1}\right) .
$$

We shall see in the next section that (2.8) stabilizes to the spectral sequence of (1.4).

\section{Stabilization}

Given a ring $R$ and a pointed simplicial set $X$., we have the simplicial abelian group $R(X$.$) with$

$$
\pi_{n}|R(X .)|=\tilde{H}_{n}(|X .| ; R) .
$$

In particular, $R\left(S^{x}\right)$ is a simplicial model of the Eilenberg-Mac Lane space of type $(R, x)$. The obvious map

$$
S^{1} \wedge R\left(S_{.}^{x}\right) \rightarrow R\left(S^{x+1}\right)
$$

gives $R\left(S^{x}\right)$ the structure of a simplicial spectrum, in fact the structure of a simplicial $\Omega$-spectrum. Indeed, the simplicial mapping space

$$
\begin{aligned}
\Omega . R\left(S_{.}^{x+1}\right) & =\operatorname{s.sets}\left(S_{\cdot}^{1}, R\left(S_{.}^{x+1}\right)\right) \\
& =\operatorname{s.Ab}\left(\mathbf{Z}\left(S_{\cdot}^{1}\right), R\left(S^{x+1}\right)\right) \\
& \cong R\left(S^{x}\right) .
\end{aligned}
$$

Here $\operatorname{s.} \operatorname{Ab}\left(A_{.}, B\right.$. $)$ denotes the simplicial mapping space in the category of simplicial abelian groups and $\operatorname{s.sets}(X, Y$.$) the same in the category of pointed simplicial$ sets. The equivalence is from [Q], but of course also follows from the fact that the 
realizations of simplicial mapping spaces are the mapping spaces of the realizations when the target is fibrant (which simplicial abelian groups are), and the homotopical uniqueness of Eilenberg-Mac Lane spectra.

Given a pair $M, N$ of a right and a left $R$-module, we have the corresponding endofunctors of simplicial sets of (2.3), and the functor

$$
X . \mapsto \mathcal{B} .(M, R, N)(X .)
$$

of (2.2). The stabilization maps $X . \wedge M(Y.) \rightarrow M(X . \wedge Y$.), etc., induce a map

$$
X . \wedge \mathcal{B} .(M, R, N)(Y .) \rightarrow \mathcal{B} .(M, R, N)(X . \wedge Y \text {. })
$$

and in particular a map of realizations

$$
S^{1} \wedge \mathcal{B}(M, R, N)\left(S^{x}\right) \rightarrow \mathcal{B}(M, R, N)\left(S^{x+1}\right),
$$

so that $\mathcal{B}(M, R, N)\left(S^{x}\right), x=0,1, \ldots$, becomes a prespectrum. Note of course that $\mathcal{B}(M, R, N)\left(S^{x}\right)$ is more complicated than just the realization of the free simplicial object on $S^{x}$ over the simplicial abelian group $B .(M, R, N)$. Nevertheless, we have

Lemma 3.1. The adjoints of the suspensions (3.2) are homotopy equivalences, so that $\mathcal{B}(M, R, N)\left(S^{x}\right)$ form an $\Omega$-spectrum.

Proof. We remarked in section 2 that $B(M, R, N)=\mathcal{B}(M, R, N)\left(S^{0}\right)$ has homotopy groups $\operatorname{Tor}_{*}^{R}(M, N)$. Hence it suffices to show that

$$
T_{i}(M)=\pi_{i+x} \mathcal{B}(M, R, N)\left(S^{x}\right)
$$

satisfies the axioms for the left derived functors of the functors $-\otimes_{R} N$.

When $i>0, T_{i}(M)$ vanishes for $M=R$, hence for free $R$-modules by the obvious additivity property. For $0 \rightarrow M_{1} \rightarrow M_{2} \rightarrow M_{3} \rightarrow 0$ exact,

$$
\mathcal{B} .\left(M_{1}, R, N\right)\left(S_{.}^{x}\right) \rightarrow \mathcal{B} .\left(M_{2}, R, N\right)\left(S^{x}\right) \rightarrow \mathcal{B} .\left(M_{3}, R, N\right)\left(S^{x}\right)
$$

is a degreewise exact sequence of bisimplicial abelian groups. Hence its topological realization is a fibration, and we get the desired exact sequence of homotopy groups. Third,

$$
T_{0}(M)=\pi_{x} \mathcal{B}(M, R, N)\left(S^{x}\right)=\operatorname{coker}\left(d_{0}-d_{1}: \pi_{x} M R N\left(S^{x}\right) \rightarrow \pi_{x}\left(M N\left(S^{x}\right)\right)\right),
$$

where $M N\left(S^{x}\right)=M\left(N\left(S^{x}\right)\right)$, etc., and

$$
\pi_{x} M N\left(S^{x}\right) \cong H_{x}\left(N\left(S^{x}\right) ; M\right) \simeq H_{x}\left(N\left(S^{x}\right) ; \mathbf{Z}\right) \otimes M
$$

since $N\left(S^{x}\right)$ is $(x-1)$-connected. This connectivity implies also that

$$
H_{x}\left(N\left(S^{x}\right) ; \mathbf{Z}\right) \cong \pi_{x} N\left(S^{x}\right) \cong H_{x}\left(S^{x} ; N\right),
$$

and it follows that

$$
\pi_{x} M N\left(S^{x}\right)=M \otimes \mathbf{z} N
$$

Similarly,

$$
\pi_{x} M R N\left(S^{x}\right)=M \otimes_{Z} R \otimes_{Z} N
$$

and $T_{0}(M)$ becomes the coequalizer of

$$
M \otimes_{Z} R \otimes_{Z} N \rightrightarrows M \otimes_{\mathbf{z}} N
$$

This is by definition $M \otimes_{R} N$. We have verified the standard axioms for left derived functors, and conclude that

$$
T_{i}(M) \cong \operatorname{Tor}_{i}^{R}(M, N) .
$$


Finally, it is quite obvious that the adjoints of $(3.2)$ induce the isomorphisms.

Next, we will stabilize the construction $W .(M, R, N)$. Following Bökstedt we let $I$ denote the (skeleton of the) category of finite sets and injective maps: the objects are the non-negative integers, and the set of morphisms from $x$ to $y$, for $x \leq y$, is the set of inclusions.

Given $\mathbf{x}=\left(x_{0}, \ldots, x_{k+1}\right) \in I^{k+2}$, let

$$
\begin{aligned}
& W_{k}(M, \mathbf{Z}(R), \mathbf{Z}(N))(\mathbf{x}) \\
& =M\left(S_{\cdot}^{x_{0}}\right) \otimes \mathbf{Z}\left(R\left(S_{\cdot}^{x_{1}}\right)\right) \otimes \cdots \otimes \mathbf{Z}\left(R\left(S_{\cdot}^{x_{k}}\right)\right) \otimes \mathbf{Z}\left(N\left(S_{\cdot}^{x_{k+1}}\right)\right) \\
& W_{k}(M, \mathbf{Z}(R), \mathbf{Z}(N) ; \mathbf{x}) \\
& \quad=\operatorname{s.Ab}\left(\mathbf{Z}\left(S^{x_{0}} \wedge \cdots \wedge S^{x_{k+1}}\right), W_{k}(M, \mathbf{Z}(R), \mathbf{Z}(N))(\mathbf{x})\right) .
\end{aligned}
$$

The concatenation map $d_{i}: I^{k+2} \rightarrow I^{k+1}$ which adds $x_{i}$ and $x_{i+1}$ induces maps

$$
\begin{aligned}
\mathbf{Z}\left(S^{x_{i}} \wedge S^{x_{i+1}}\right) & \rightarrow \mathbf{Z}\left(S^{x_{i}+x_{i+1}}\right), \\
\mathbf{Z}\left(R\left(S^{x_{i}}\right)\right) \otimes \mathbf{Z}\left(R\left(S^{x_{i+1}}\right)\right) & \rightarrow \mathbf{Z}\left(R\left(S^{x_{i}+x_{i+1}}\right)\right),
\end{aligned}
$$

and yields face maps

$$
d_{i}: W_{k}(M, \mathbf{Z}(R), \mathbf{Z}(N) ; \mathbf{x}) \rightarrow W_{k-1}\left(M, \mathbf{Z}(R), \mathbf{Z}(N) ; d_{i}(\mathbf{x})\right)
$$

Similarly the inclusion $s_{i}: I^{k+1} \rightarrow I^{k+2}$ which inserts 0 in the $i$ 'th place induces

$$
s_{i}: W_{k-1}(M, \mathbf{Z}(R), \mathbf{Z}(N) ; \mathbf{x}) \rightarrow W_{k}\left(M, \mathbf{Z}(R), \mathbf{Z}(N) ; s_{i}(\mathbf{x})\right) .
$$

A map $\phi=\left(\phi_{0}, \ldots, \phi_{k+1}\right)$ from $\mathbf{x}$ to $\mathbf{y}$ (in $\left.I^{k+2}\right)$ induces a map from $W_{k}(M, \mathbf{Z}(R), \mathbf{Z}(N) ; \mathbf{x})$ into $W_{k}(M, \mathbf{Z}(R), \mathbf{Z}(N) ; \mathbf{y})$, obtained by tensoring domain and range by $\mathbf{Z}\left(S^{y_{0} \backslash \phi_{0}\left(x_{0}\right)} \wedge \cdots \wedge S^{y_{k+1} \backslash \phi_{k+1}\left(x_{k+1}\right)}\right)$ and using the obvious products

$$
\begin{aligned}
& M\left(S^{x_{i}}\right) \otimes \mathbf{Z}\left(S^{y_{i} \backslash \phi_{i}\left(x_{i}\right)}\right) \cong M\left(S^{\phi_{i}\left(x_{i}\right)}\right) \otimes \mathbf{Z}\left(S^{y_{i} \backslash \phi_{i}\left(x_{i}\right)}\right) \rightarrow M\left(S^{y_{i}}\right), \\
& \mathbf{Z}\left(A\left(S^{x_{i}}\right)\right) \otimes \mathbf{Z}\left(S^{y_{i} \backslash \phi_{i}\left(x_{i}\right)}\right) \quad \rightarrow \mathbf{Z}\left(A\left(S^{x_{i}}\right) \wedge S^{y_{i} \backslash \phi_{i}\left(x_{i}\right)}\right) \quad \rightarrow \mathbf{Z}\left(A\left(S^{y_{i}}\right)\right)
\end{aligned}
$$

for $A=R, N$. We can take the homotopy direct limit over $I^{k+2}$,

$$
\mathbf{W}_{k}(M, R, N)=\underset{I^{k+2}}{\operatorname{holim}} W_{k}(M, \mathbf{Z}(R), \mathbf{Z}(N) ; \mathbf{x}) .
$$

This defines a simplicial abelian group W. $(M, R, N)$. Similarly, we set

$$
\mathbf{B}(M, R, N)=\underset{I^{k+2}}{\operatorname{holim}} \operatorname{s.Ab}\left(\mathbf{Z}\left(S^{x_{0}} \wedge \cdots \wedge S^{x_{k+1}}\right), \mathcal{B}(M, R, N)\left(S_{.}^{x_{0}} \wedge \cdots \wedge S^{x_{k+1}}\right)\right)
$$

to obtain a simplicial abelian group B. $(M, R, N)$. There is a commutative diagram

$$
\begin{array}{ccc}
W .(M, \mathbf{Z}(R), \mathbf{Z}(N)) & \stackrel{\mathrm{ev}}{\longrightarrow} & B .(M, R, N) \\
\downarrow & & \downarrow \Sigma_{W} \\
\mathbf{W} .(M, R, N) & \stackrel{\text { ev }}{\longrightarrow} & \text { B. }(M, R, N)
\end{array}
$$

The upper horizontal map ev is the one defined in (2.7); the lower map ev is defined similarly. The vertical maps are the inclusions of the initial terms $(\mathbf{x}=(0, \ldots, 0))$ into the homotopy direct limits.

Lemma 3.2. In diagram (3.5), the lower horizontal map ev and the right-hand vertical map $\Sigma_{B}$ are homotopy equivalences. 
Proof. The homotopy direct limits are well-behaved in the sense that a finite stage approximates the limit; cf. [M], lemma 2.3.7. Thus lemma 3.1 implies that $\Sigma_{B}$ is a homotopy equivalence. To see that $\mathbf{e v}$ is a homotopy equivalence it suffices to check that it is a degreewise homotopy equivalence. This in turn is a consequence of the fact that the stabilizations of the form

$$
M\left(S^{x}\right) \wedge Y . \rightarrow M\left(S^{x} \wedge Y_{.}\right)
$$

for any abelian group induce isomorphisms on homotopy groups $\pi_{i+x}$ for $i<x$. Indeed,

$$
\pi_{i+x}\left(M\left(S^{x}\right) \wedge Y\right) \cong \tilde{H}_{i+x}\left(S^{x} \wedge Y ; M\right), \quad i<x,
$$

by the standard relationship between singular homology and homology defined in terms of spectra. Moreover, formula (3.1) gives

$$
\tilde{H}_{i+x}\left(S^{x} \wedge Y ; M\right)=\pi_{i+x}\left(M\left(S^{x} \wedge Y\right)\right)
$$

We note as a consequence of equation (2.4) and lemma 3.2 that

$$
\pi_{n} \mathbf{W}(M, R, N)=\operatorname{Tor}_{n}^{R}(M, N)
$$

The stabilization from $W .(M, \mathbf{Z}(R), \mathbf{Z}(N))$ to $\mathbf{W} .(M, R, N)$ can also be applied to the simplicial abelian group $V .(\mathbf{Z}(R), M)$ with $k$-simplices

$$
V_{k}(\mathbf{Z}(R), M)=M \otimes \mathbf{Z}(R)^{\otimes k}
$$

where $M$ is an $R$ (and hence a $\mathbf{Z}(R)$ ) bimodule. Indeed, for $\mathbf{x} \in I^{k+1}$ one defines

$$
\begin{aligned}
V_{k}(\mathbf{Z}(R), M)(\mathbf{x}) & =M\left(S^{x_{0}}\right) \otimes \mathbf{Z} R\left(S^{x_{1}}\right) \otimes \cdots \otimes \mathbf{Z} R\left(S^{x_{k}}\right), \\
V_{k}(\mathbf{Z}(R), M ; \mathbf{x}) & =\operatorname{s.Ab}\left(\mathbf{Z}\left(S^{x_{0}} \wedge \cdots \wedge S^{x_{k}}\right), V_{k}(\mathbf{Z}(R), M)(\mathbf{x})\right),
\end{aligned}
$$

and sets

$$
\mathbf{V}_{k}(R, M)=\underset{I^{k+1}}{\operatorname{holim}} \operatorname{s.Ab}\left(\mathbf{Z}\left(S_{\cdot}^{x_{0}}\right) \otimes \cdots \otimes \mathbf{Z}\left(S_{\cdot}^{x_{k}}\right), V_{k}(\mathbf{Z}(R), M)(\mathbf{x})\right) .
$$

This defines a simplicial abelian group $\mathbf{V} .(R, M)$ and in fact a model for $\operatorname{THH}(R, M)$ :

$$
\pi_{n} \mathbf{V}(R, M)=\mathrm{THH}_{n}(R, M)
$$

cf. [DM1] or [M], (3.2.8). There are simplicial maps

$$
\begin{aligned}
& \Sigma_{V}: V .(\mathbf{Z}(R), M) \rightarrow \mathbf{V} .(R, M) \quad \text { (stabilization), } \\
& L_{V}: \mathbf{V} .(R, M) \rightarrow V .(R, M) \quad \text { (linearization). }
\end{aligned}
$$

The linearization $L_{V}$ is the map that replaces the simplicial set of $k$-simplices $\mathbf{V}_{k}(R, M)$ with its group of components $\pi_{0}\left(\mathbf{V}_{k}(R, M)\right)=V_{k}(R, M)$. On homotopy groups we get

$$
\mathrm{HH}_{*}(\mathbf{Z}(R), M) \stackrel{\Sigma}{\rightarrow} \mathrm{THH}_{*}(R, M) \stackrel{L}{\rightarrow} \mathrm{HH}_{*}(R, M) .
$$

The composition $L \circ \Sigma$ is the homomorphism of Hochschild homology induced by the ring homomorphism $\mathbf{Z}(R) \rightarrow R$.

The topological Hochschild homology $\operatorname{THH}(R, M)$ is the zero'th space of a spectrum $\operatorname{TH}(R, M)$, and there is a pairing

$$
\mathrm{TH}(R, M) \wedge \mathrm{TH}\left(R^{\prime}, M^{\prime}\right) \rightarrow \mathrm{TH}\left(R \otimes R^{\prime}, M \otimes M^{\prime}\right)
$$


of spectra (cf. [HM], sect. 2.7). In the special case where $\pi: R \rightarrow R_{1}$ is a homomorphism of commutative rings, the composition

$$
\mathrm{TH}\left(R, R_{1}\right) \wedge \mathrm{TH}\left(R, R_{1}\right) \rightarrow \mathrm{TH}\left(R \otimes R, R_{1} \otimes R_{1}\right) \rightarrow \mathrm{TH}\left(R, R_{1}\right)
$$

makes $\mathrm{TH}\left(R, R_{1}\right)$ into a ring spectrum, and in particular induces a graded ring structure on the homotopy groups $\operatorname{THH}_{*}\left(R, R_{1}\right)$.

Theorem 3.3 (M. Brun). Let $R \stackrel{\pi}{\rightarrow} R_{1}$ be a homomorphism of rings. There is a homology type first quadrant spectral sequence

$$
\begin{aligned}
& E_{k, l}^{2}\left(R \rightarrow R_{1}\right)=\operatorname{THH}_{k}\left(R_{1}, \operatorname{Tor}_{\ell}^{R}\left(R_{1}, R_{1}\right)\right) \\
& E_{*, *}^{\infty}\left(R \rightarrow R_{1}\right)=\operatorname{THH}_{*}\left(R, R_{1}\right) .
\end{aligned}
$$

If $R$ and $R_{1}$ are commutative, then $E_{*, *}^{r}$ is a ring and the differential $d^{r}$ is a derivation in the usual bigraded sense.

Proof (sketch). The proof we indicate is an obvious variant of Morten Brun's argument; he used a different model for THH. The reader should note the similarity with the spectral sequence (2.8).

For $\mathbf{x} \in I^{k}$ and $\mathbf{y} \in I^{\ell+2}$ consider the simplicial abelian group

$$
\begin{aligned}
& V_{k}\left(\mathbf{Z}\left(R_{1}\right), W_{\ell}\left(R_{1}, \mathbf{Z}(R), \mathbf{Z}\left(R_{1}\right)\right)(\mathbf{y})\right)(\mathbf{x}) \\
& \quad=R_{1}\left(S^{y_{0}}\right) \otimes \mathbf{Z} R\left(S^{y_{1}}\right) \otimes \cdots \otimes \mathbf{Z} R\left(S^{y_{\ell}}\right) \otimes \mathbf{Z} R_{1}\left(S^{y_{\ell+1}}\right) \otimes \mathbf{Z} R_{1}\left(S^{x_{1}}\right) \otimes \cdots \otimes \mathbf{Z} R_{1}\left(S^{x_{k}}\right) .
\end{aligned}
$$

We can apply the functor

$$
\mathrm{s.Ab}\left(\bigotimes \mathbf{Z}\left(S_{\cdot}^{x_{i}}\right) \otimes \bigotimes \mathbf{Z}\left(S^{y_{j}}\right),-\right)
$$

and take the homotopy direct limit over $\mathbf{x} \in I^{k}$ and $\mathbf{y} \in I^{\ell+2}$ to get a simplicial abelian group which we denote $T_{k, \ell}\left(R \rightarrow R_{1}\right)$. Letting $k$ and $\ell$ vary, we get a bisimplicial object in the category of simplicial abelian groups with

$$
T_{0, .}\left(R \rightarrow R_{1}\right)=\mathbf{W} .\left(R_{1}, R, R_{1}\right) .
$$

We can diagonalize the $\ell$-direction and the simplicial direction to obtain a bisimplicial abelian group, hence a bicomplex whose associated spectral sequence has

$$
E_{k, \ell}^{1}\left(R \rightarrow R_{1}\right) \cong \mathbf{V}_{k}\left(R_{1}, \pi_{\ell} \mathbf{W}\left(R_{1}, R, R_{1}\right)\right),
$$

and thus

$$
E_{k, \ell}^{2}=\pi_{k}\left(\mathbf{V}\left(R_{1}, \pi_{\ell}\left(\mathbf{W}\left(R_{1}, R, R_{1}\right)\right)\right)\right)=\operatorname{THH}_{k}\left(R_{1}, \operatorname{Tor}_{\ell}^{R}\left(R_{1}, R_{1}\right)\right) .
$$

On the other hand, we can rearrange factors as in the proof of (2.8) and use the fact that each

$$
W .\left(\mathbf{Z}\left(R_{1}\right), \mathbf{Z}\left(R_{1}\right), R_{1}\right) \simeq R_{1}
$$

to show that the total complex of $T_{., .}\left(R \rightarrow R_{1}\right)$ calculates $\mathrm{THH}_{*}\left(R, R_{1}\right)$.

The reader can consult Brun1 to check that we get a spectral sequence of graded algebras in the commutative situation.

The model for $\operatorname{THH}(R, M)$ used above does not have the property that $\mathbf{V}$. $(R, R)$ is a cyclic simplicial set, so one does not see the $S^{1}$-action on $\operatorname{THH}(R, R)$ in this model. However, $\mathbf{V} .(R, M)$ is very convenient when one compares with the linear situation. In particular, there is a stabilization map

$$
\Sigma: V .\left(\mathbf{Z}\left(R_{1}\right), W .\left(R_{1}, \mathbf{Z}(R), \mathbf{Z}\left(R_{1}\right)\right) \rightarrow T_{., .}\left(R \rightarrow R_{1}\right)\right.
$$


that induces a map from the spectral sequence of (2.8) to the spectral sequence of theorem 3.3 The map $\Sigma$ is the composition of two stabilization maps: one can first stabilize $W$. to $\mathbf{W}$., then $V$. to $\mathbf{V}$., and $\Sigma=\Sigma_{V} \circ \Sigma_{W}$. On the $E^{2}$-terms we get induced homomorphisms

$$
\begin{gathered}
\mathrm{HH}_{k}\left(\mathbf{Z}\left(R_{1}\right), \operatorname{Tor}_{\ell}^{\mathbf{Z}(R)}\left(R_{1}, Z\left(R_{1}\right)\right)\right) \longrightarrow \underset{W}{\operatorname{HH}_{k}\left(\mathbf{Z}\left(R_{1}\right), \operatorname{Tor}_{\ell}^{R}\left(R_{1}, R_{1}\right)\right)} \\
\operatorname{THH}_{k}\left(R_{1}, \operatorname{Tor}_{\ell}^{R}\left(R_{1}, R_{1}\right)\right)
\end{gathered}
$$

Alternatively (and this is what we use in the next section), by 3.5) one can first use

$$
e: W .\left(R_{1}, \mathbf{Z}(R), \mathbf{Z}\left(R_{1}\right)\right) \rightarrow B .\left(R_{1}, R, R_{1}\right)
$$

to get into the bisimplicial set $V .\left(\mathbf{Z}\left(R_{1}\right), B .\left(R_{1}, R, R_{1}\right)\right)$. Consider the diagram

$$
\begin{gathered}
V .\left(\mathbf{Z}\left(R_{1}\right), \underset{.}{B .}\left(R_{1}, R, R_{1}\right)\right) \quad \stackrel{\Sigma_{V}}{\longrightarrow} \quad \mathbf{V} .\left(R_{1}, B .\left(R_{1}, R, R_{1}\right)\right) \\
V .\left(R_{1}, B .\left(R_{1}, R, R_{1}\right)\right)
\end{gathered}
$$

with $\mu: \mathbf{Z}\left(R_{1}\right) \rightarrow R_{1}$ the evaluation ring homomorphism. The diagram 3.11 induces a diagram of spectral sequences, namely of the spectral sequences of the associated double complexes. By (3.5) and Lemma 3.2, the upper right-hand corner is homotopy equivalent to the $T_{. . .}\left(R \rightarrow R_{1}\right)$, so its spectral sequence is that of Theorem 3.3. On the $E^{2}$-terms we have

$$
\begin{gathered}
\mathrm{HH}_{*}\left(\mathbf{Z}\left(R_{1}\right), \operatorname{Tor}_{*}^{R}\left(R_{1}, R_{1}\right)\right) \stackrel{\Sigma_{*}}{\longrightarrow} \operatorname{THH}_{*}\left(R_{1}, \operatorname{Tor}_{*}^{R}\left(R_{1}, R_{1}\right)\right) \\
\underset{H_{*}}{\longrightarrow}\left(R_{1}, \operatorname{Tor}_{*}^{R}\left(R_{1}, R_{1}\right)\right) .
\end{gathered}
$$

\section{Differentials}

In this section $A$ is a characteristic zero complete discrete valuation ring with perfect residue field $k$ of characteristic $p>0$.

We calculate the spectral sequence $E_{*, *}^{r}(A \rightarrow A / p)$ of theorem 3.3 . The resolution $0 \rightarrow A \stackrel{p}{\rightarrow} A \rightarrow A / p \rightarrow 0$ tells us that

$$
\operatorname{Tor}_{0}^{A}(A / p, A / p)=A / p=\operatorname{Tor}_{1}^{A}(A / p, A / p),
$$

and that the higher Tor-groups vanish. Thus the spectral sequence is concentrated on two lines,

$$
\begin{aligned}
& E_{*, 0}^{2}(A \rightarrow A / p)=\operatorname{THH}_{*}(A / p), \\
& E_{*, 1}^{2}(A \rightarrow A / p)=\mathrm{THH}_{*}(A / p) \cdot \tau,
\end{aligned}
$$

where $\tau \in E_{0,1}^{2}(A \rightarrow A / p)$, and thus the only possible differentials are the $d^{2}$ differentials from $E_{r, 0}^{2}$ to $E_{r-2,1}^{2}$.

We need to recall some terminology and some facts about $A$, all of which can be found in [S], Chapters II and III.

Let $A_{0}=W(k)$ denote the Witt vectors of $k$. Then $A_{0}$ is a subring of $A$, and $A$ is free over $A_{0}$ of rank $e$, the total ramification index. Let $\pi \in A$ be a uniformizer. Then

$$
\pi^{e}=p u, u \in A^{\times} ; \quad A / p=k[\pi] /\left(\pi^{e}\right) .
$$


The element $\pi$ satisfies an Eisenstein polynomial $\phi$ over $A_{0}$ of degree $e$,

$$
A=A_{0}[x] /(\phi(x)) .
$$

It is useful to divide the calculation into cases in the following standard fashion:

(i) $A$ is unramified if $e=1$.

(ii) $A$ is wildly ramified if $p$ divides $e$.

(iii) $A$ is tamely ramified if $e>1$ is coprime to $p$.

The topological Hochschild homology of $A / p$ was calculated in [HM], sect. 7, or in $[\mathrm{L}]$ when $k=\mathbf{F}_{p}$. The result is

$$
\mathrm{THH}_{*}(A / p) \cong S_{k}\{\sigma\} \otimes_{k} \operatorname{HH}_{*}(A / p), \operatorname{deg} \sigma=2 .
$$

Under this isomorphism, linearization

$$
L: \operatorname{THH}_{*}(A / p) \rightarrow \mathrm{HH}_{*}(A / p)
$$

corresponds to augmentation $S_{k}\{\sigma\} \rightarrow k ; S_{k}\{\sigma\}$ denotes the polynomial algebra over $k$.

We will also need the values of $\mathrm{HH}_{*}(A / p)$ explicitly. Let $E_{A / p}\left\{c_{1}\right\}$ denote the exterior algebra over $A / p$ on a one-dimensional generator, and let $\Gamma_{A / p}\left\{c_{2}\right\}$ be the divided polynomial algebra on a two-dimensional generator, i.e., the free graded $A / p$-module on generators $\gamma_{i}\left(c_{2}\right)$ of degree $2 i$ with

$$
\gamma_{i}\left(c_{2}\right) \gamma_{j}\left(c_{2}\right)=\left(\begin{array}{c}
i+j \\
i
\end{array}\right) \gamma_{i+j}\left(c_{2}\right) .
$$

Proposition 4.1. The algebra $\mathrm{HH}_{*}(A / p)$ in the three cases of (4.3) is respectively

(i) $\mathrm{HH}_{*}(A / p)=0$ for $*>0, \mathrm{HH}_{0}(A / p)=A / p \quad$ (A unramified),

(ii) $\mathrm{HH}_{*}(A / p)=\Gamma_{A / p}\left\{c_{2}\right\} \otimes_{A} E_{A / p}\left\{c_{1}\right\} \quad$ (A wildly ramified),

(iii) $\mathrm{HH}_{*}(A / p)=\pi \Gamma_{A / p}\left\{c_{2}\right\} \oplus c_{1} \cdot \Gamma_{A / \pi^{e-1}}\left\{c_{2}\right\} \quad$ (A tamely ramified).

The algebra structure in part (iii) is to be interpreted as that coming from the inclusion into $\Gamma_{A / p}\left\{c_{2}\right\} \otimes_{A} E_{A / p}\left\{c_{1}\right\} /\left(c_{1} \pi^{e-1}\right)$.

The proof of Proposition 4.1 is based on results from [BAG, which we first review. Let $\Lambda$ be a commutative ring, and $A=\Lambda[x] /(\phi(x))$ for some monic polynomial. Consider $A$ as an $A^{e}=A \otimes_{\Lambda} A$ module, and let $C_{*}^{\Lambda}(A) \rightarrow A$ be the free $A^{e}$-resolution with each $C_{i}(A)$ free of rank 1 ; if $c_{i}$ is the generator in degree $i$ then

$$
\begin{aligned}
\partial c_{2 n} & =\frac{\phi(x) \otimes 1-1 \otimes \phi(x)}{x \otimes 1-1 \otimes x} \cdot c_{2 n-1}, \\
\partial c_{2 n-1} & =(x \otimes 1-1 \otimes x) \cdot c_{2 n-2} .
\end{aligned}
$$

Let

$$
Z_{*}^{\Lambda}(A, M)=M \otimes_{A^{e}} C_{*}^{\Lambda}(A) .
$$

The homology of $\mathbf{Z}_{*}^{\Lambda}(A, M)$ is the Hochschild homology $\operatorname{HH}_{*}^{\Lambda}(A, M)$ of $A$ with respect to the ground ring $\Lambda$. Indeed, $W_{*}^{\Lambda}(A, A, A)$, defined like $W$ but with tensors taken over $\Lambda$, and $C_{*}^{\Lambda}(A)$ are both free $\Lambda$-resolutions of $A$, so tensoring with $M$ one gets chain homotopy equivalences

$$
Z_{*}^{\Lambda}(A, M) \rightleftarrows V_{*}^{\Lambda}(A, M) .
$$


(These are made explicit in $[\mathrm{BAG}]$ in terms of the coefficients of $\phi(x)$.) We note, when the $A^{e}$ module $M$ comes from an $A$ module structure, that $Z_{*}^{\Lambda}(A, M)$ reduces to the chain complex

$$
\cdots \stackrel{\phi^{\prime}(x)}{\longrightarrow} M \stackrel{0}{\longrightarrow} M \stackrel{\phi^{\prime}(x)}{\longrightarrow} M \stackrel{0}{\longrightarrow} \cdots \stackrel{0}{\longrightarrow} M \longrightarrow 0 .
$$

In particular, (4.2) yields

$$
\mathrm{HH}_{2 n-1}^{A_{0}}(A)=A / \mathcal{D}_{A} \cong \mathcal{D}_{A}^{-1} / A, \quad \mathrm{HH}_{2 n}^{A_{0}}(A)=0 \text { for } n>0,
$$

and $\operatorname{HH}_{0}^{A_{0}}(A)=A$.

Proof of Proposition 4.1. We note first that the natural map

$$
\mathrm{HH}_{*}(A / p) \rightarrow \mathrm{HH}_{*}^{A_{0}}(A / p)
$$

is an isomorphism. If $A=A_{0}$, this is the statement that $\mathrm{HH}_{*}(k)=0$ for $*>0$ for a perfect field $k$ (see e.g. $[\mathrm{HM}$, lemma 5.5). For general $A$, it follows from the change of rings spectral sequence

$$
\operatorname{Tor}^{\mathrm{HH}_{*}\left(A_{0} / p\right)}\left(\mathrm{HH}_{*}(A / p), A_{0} / p\right) \Rightarrow \operatorname{HH}_{*}^{A_{0}}(A / p)
$$

or directly from (4.1). The additive structure of $\operatorname{HH}_{*}^{A_{0}}(A / p)=\mathrm{HH}_{*}^{A_{0}}(A, A / p)$ follows from (4.8) and the values of $\mathcal{D}_{A / A_{0}}$ listed in (5.3). The multiplicative structure is a consequence of the $A^{e}$ chain map

$$
\begin{aligned}
m_{*}\left(c_{2 n-2 i} \otimes c_{2 i}\right) & =\left(\begin{array}{c}
n \\
i
\end{array}\right) c_{2 n}, \\
m_{*}\left(c_{2 n-2 i+1} \otimes c_{2 i}\right) & =m_{*}\left(c_{2 n-2 i} \otimes c_{2 i+1}\right)=\left(\begin{array}{c}
n \\
i
\end{array}\right) c_{2 n+1}, \\
m_{*}\left(c_{2 i+1} \otimes c_{2 j+1}\right) & =0 .
\end{aligned}
$$

Indeed, this $m_{*}$ lifts the multiplication $A \otimes_{A_{0}} A \rightarrow A$.

We shall now make use of the structure of $A / p$ given in (4.1). Consider the pointed monoid

$$
\Pi_{e}=\left\{0,1, \pi, \ldots, \pi^{e-1}\right\}, \quad \pi^{e}=0 .
$$

The inclusion of $\Pi_{e}$ in $A / p$ extends to an identification $k\left(\Pi_{e}\right)=A / p$, and shows that the natural homomorphism

$$
\mu: k(A / p) \rightarrow A / p
$$

is a split surjection of rings; a splitting is given by $\nu=k(\iota)$, where $\iota: \Pi_{e} \rightarrow A / p$ is the inclusion. This induces a simplicial map

$$
V .(\nu): V .(A / p, B .(A / p, A, A / p)) \rightarrow V .(\mathbf{Z}(A / p), B .(A / p, A, A / p)) .
$$

Indeed, $B .=B .(A / p, A, A / p)$ is a $k$-module, and thus

$$
V .(\mathbf{Z}(A / p), B .)=B . \otimes_{\mathbf{Z}} \mathbf{Z}(A / p)^{(r)}=B . \otimes_{k} k(A / p)^{(r)},
$$

where $(r)$ indicates the $r$-fold tensor product over $k$. From (3.11) we get the commutative diagram of bisimplicial abelian groups

$$
\begin{gathered}
V .(A / p, B .(A / p, A, A / p)) \quad \stackrel{\Sigma \circ V_{.}(\nu)}{\longrightarrow} \quad \mathbf{V} .(\mathbf{Z}(A / p), B .(A / p, A, A / p)) \\
\qquad \begin{array}{l}
\text { id } \\
V .(A / p, B .(A / p, A, A / p))
\end{array}
\end{gathered}
$$


and a resulting splitting of the associated spectral sequences that on the $E^{2}$ level becomes the triangle

$$
\begin{gathered}
\operatorname{HH}_{*}\left(A / p, \operatorname{Tor}_{*}^{A}(A / p, A / p)\right) \stackrel{\nu_{*}}{\longrightarrow} \underset{\mathrm{id}}{\operatorname{THH}}\left(A / p, \operatorname{Tor}_{*}^{A}(A / p, A / p)\right) \\
\operatorname{HH}_{*}\left(A / p, \operatorname{Tor}_{*}^{A}(A / p, A / p)\right)
\end{gathered}
$$

of bigraded algebra homomorphisms which commute with $d^{2}$. These spectral sequences are each concentrated on two (isomorphic) horizontal lines, and the fiber lines $E_{0, *}^{2}$ are the same in both. Let $\tau \in E_{0,1}^{2} \cong A / p$ be the generator corresponding to $1 \in A / p$.

Proposition 4.2. For the top left-hand spectral sequence in (4.13), we have:

(i) For A wildly ramified,

$$
d^{2}\left(\gamma_{r}\left(c_{2}\right)\right)=u \gamma_{r-1}\left(c_{2}\right) \tau, \quad d^{2}\left(\gamma_{r}\left(c_{2}\right) c_{1}\right)=u \gamma_{r-1}\left(c_{2}\right) c_{1} \tau
$$

(ii) For A tamely ramified,

$$
d^{2}\left(\pi \gamma_{r}\left(c_{2}\right)\right)=u \pi \gamma_{r-1}\left(c_{2}\right) \tau, \quad d^{2}\left(\gamma_{r}\left(c_{2}\right) c_{1}\right)=u \gamma_{r-1}\left(c_{2}\right) c_{1} \tau
$$

Here we have used the notation of Proposition 4.1, with $u$ defined by (4.1).

Proof. We begin by exhibiting a specific map $f_{*}$ from the standard $A$-free resolution $B_{*}(A, A, A / p)$ of $A / p$ into the resolution

$$
0 \rightarrow A \stackrel{p}{\rightarrow} A \rightarrow A / p \rightarrow 0
$$

i.e., we need $f_{0}, f_{1}$ in

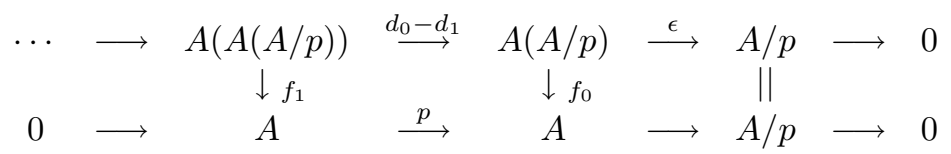

Choose a pointed set map $\bar{s}: k \rightarrow A$ which splits the natural projection from $A$ to $k=A / \pi$ so that $\bar{s}(1+(\pi))=1$, and let

$$
s: k\left(\Pi_{e}\right) \rightarrow A ; \quad s\left(\sum_{i=0}^{e-1} \lambda_{i} \pi^{i}\right)=\sum_{i=0}^{e-1} \bar{s}\left(\lambda_{i}\right) \pi^{i} .
$$

We take $f_{0}=\mu \circ A(s)$, where $\mu: A(A) \rightarrow A$ evaluates a formal linear combination, and let $f_{1}=\frac{1}{p}\left(f_{0} \circ\left(d_{0}-d_{1}\right)\right)$. We denote elements of $B_{n}(A, A, A / p)$ by $a_{0} \circ \cdots \circ a_{n}$, $a_{i} \in A$ for $i<n$ and $a_{n} \in A / p$; the expression is linear in $a_{0}$ but not in the other variables, and it is zero if any of the variables is zero. We shall need the formula

$$
f_{1}\left(\pi^{a} \circ \pi^{b} \circ \pi^{c}\right)= \begin{cases}0 & \text { if } b+c<e, \\ u \pi^{a+b+c-e} & \text { if } b+c \geq e,\end{cases}
$$

where $u \in A^{\times}$is the unit from formula (4.1). Indeed,

$$
d_{0}\left(\pi^{a} \circ \pi^{b} \circ \pi^{c}\right)=\pi^{a+b} \circ \pi^{c}, \quad d_{1}\left(\pi^{a} \circ \pi^{b} \circ \pi^{c}\right)=\pi^{a} \circ \pi^{b+c} .
$$

If $b+c<e$, then

$$
f_{0} d_{0}\left(\pi^{a} \circ \pi^{b} \circ \pi^{c}\right)=\pi^{a+b+c}=f_{0} d_{1}\left(\pi^{a} \circ \pi^{b} \circ \pi^{c}\right),
$$

so $f_{1}\left(\pi^{a} \circ \pi^{b} \circ \pi^{c}\right)=0$. If $b+c \geq e$, then $\pi^{b+c}=0$ in $A / p$ and $f_{1}\left(\pi^{a} \circ \pi^{b} \circ \pi^{c}\right)=$ $1 / p \cdot \pi^{a+b+c}=u \pi^{a+b+c-e}$. 
We now use the homotopy equivalence (4.7) and replace the double complex $V_{*}\left(A / p, B_{*}(A / p, A, A / p)\right)$ by $Z_{*}\left(A / p, B_{*}(A / p, A, A / p)\right)$. Note that the right action of $A / p$ on $B_{n}(A / p, A, A / p)$ goes through the identification $A / p=k\left(\Pi_{e}\right)$,

$$
\left(a_{0} \circ \cdots \circ a_{n}\right) \cdot \sum_{i=0}^{e-1} \lambda_{i} \pi^{i}=\sum_{i=0}^{e-1} \lambda_{i} a_{0} \circ \cdots \circ a_{n} \pi^{i} .
$$

In order to calculate

$$
d^{2}: \operatorname{HH}_{r}\left(A / p, \operatorname{Tor}_{0}^{A}(A / p, A / p)\right) \rightarrow \operatorname{HH}_{r-2}\left(A / p, \operatorname{Tor}_{1}^{A}(A / p, A / p)\right),
$$

we must chase elements through the additive relation

$$
Z_{r}\left(A / p, B_{0}\right) \stackrel{\delta}{\longrightarrow} Z_{r-1}\left(A / p, B_{0}\right) \stackrel{\partial}{\longleftarrow} Z_{r-1}\left(A / p, B_{1}\right) \stackrel{\delta}{\longrightarrow} Z_{r-2}\left(A / p, B_{1}\right),
$$

where $B_{i}=B_{i}(A / p, A, A / p), \delta$ is the $Z_{*}$-differential, and $\partial$ the $B_{*}$-differential. Since $A / p=k[\pi] /\left(\pi^{e}\right)$, it follows that $\phi(x)=x^{e}$ in (4.5), and for any $b \in B_{*}$

$$
\begin{aligned}
\delta\left(b \otimes c_{2 r}\right) & =\sum_{k_{0}=0}^{e-1} \pi^{k_{0}} b \pi^{k_{1}} \otimes c_{2 r-1}, \text { where } k_{1}=e-1-k_{0}, \\
\delta\left(b \otimes c_{2 r-1}\right) & =(\pi b-b \pi) \otimes c_{2 r-2} .
\end{aligned}
$$

We can now complete the proof. In case (i), $d^{2} c_{1}=0$, so $d^{2}\left(c_{1} \gamma_{r}\left(c_{2}\right)\right)=c_{1} d^{2} \gamma_{r}\left(c_{2}\right)$. The element $\gamma_{r}\left(c_{2}\right)$ is represented by $b \otimes c_{2 r}$ with $b=1 \circ 1$ and $\pi^{k_{0}} b \pi^{k_{1}}=\pi^{k_{0}} \circ \pi^{k_{1}}$. Thus for $\partial: B_{1} \rightarrow B_{0}$ we have

$$
\partial\left(\sum_{k_{0}=1}^{e-1} \sum_{i=0}^{k_{0}-1} \pi^{k_{0}-i-1} \circ \pi \circ \pi^{k_{1}+i}\right)=\sum_{k_{0}=0}^{e-1} \pi^{k_{0}} \circ \pi^{k_{1}}-e \pi^{e-1}=\sum_{k_{0}=0}^{e-1} \pi^{k_{0}} \circ \pi^{k_{1}},
$$

since $e \equiv 0(\bmod p)$.

Thus $d^{2}\left(\gamma_{r}\left(c_{2}\right)\right)$ is represented by

$$
\begin{aligned}
& \delta\left(\sum_{k_{0}=0}^{e-1} \sum_{i=0}^{k_{0}-1} \pi^{k_{0}-i-1} \circ \pi \circ \pi^{k_{1}+i} \otimes c_{2 r-1}\right) \\
& \quad=\sum_{k_{0}=0}^{e-1} \sum_{i=0}^{k_{0}-1}\left(\pi^{k_{0}-i} \circ \pi \circ \pi^{k_{1}+i}-\pi^{k_{0}-i-1} \circ \pi \circ \pi^{k_{1}+i+1}\right) \otimes c_{2 r-2} \\
& =\sum_{k_{0}=0}^{e-1}\left(\pi^{k_{0}} \circ \pi \circ \pi^{k_{1}}-1 \circ \pi \circ \pi^{k_{0}+k_{1}}\right) \otimes c_{2 r-2} \\
& =\sum_{k_{0}=0}^{e-1} \pi^{k_{0}} \circ \pi \circ \pi^{k_{1}} \otimes c_{2 r-2}, \quad \text { as } e \equiv 0(\bmod p) .
\end{aligned}
$$

We still have to see that $\sum_{k_{0}=0}^{e-1} \pi^{k_{0}} \circ \pi \circ \pi^{k_{1}}$ represents $u \in A / p=\operatorname{Tor}_{1}^{A}(A / p, A / p)$. This follows from (4.14), and completes the proof of (i).

The proof of (ii) is entirely similar. This time we must chase the additive relation on the elements $\pi \circ 1 \otimes c_{2 r}$ and $1 \otimes c_{2 r+1}$ that represent $\pi \gamma_{r}\left(c_{2}\right)$ and $c_{1} \gamma_{r}\left(c_{2}\right)$, respectively:

$$
d^{2}\left(\gamma_{r}\left(c_{2}\right)\right)=\sum_{k_{0}=0}^{e-1} \pi^{k_{0}} \circ \pi \circ \pi^{k_{1}} \otimes c_{2 r-2}, \quad k_{1}=e-k_{0} .
$$

Use of (4.14) gives the first equation of (ii). 
For the second equation, we take $b=1 \circ 1 \in A / p(A / p)$ and consider

$$
\delta\left(1 \circ 1 \otimes c_{2 r+1}\right)=(\pi \circ 1-1 \circ \pi) c_{2 r} .
$$

Since $\partial(1 \circ \pi \circ 1)=\pi \circ 1-1 \circ \pi$

$$
d^{2}\left(1 \circ 1 \otimes c_{2 r+1}\right)=\sum_{k_{1}=0}^{e-1} \pi^{k_{0}} \circ \pi \circ \pi^{e-1-k_{0}} \otimes c_{2 r-1} .
$$

By (4.14), $f_{1}$ annihilates all summands except the one corresponding to $k_{0}=0$, which gives the stated value.

We are now ready to determine the differential of the spectral sequence $E^{r}(A \rightarrow A / p)$ of Theorem [3.3. The $E^{2}$ term is given by (4.4) and Proposition 4.1.

Proposition 4.3. The $d^{2}$ differentials in $E^{2}(A \rightarrow A / p)$ are multiplicatively generated from

(i) $d^{2} \sigma=\tau$.

(ii) For A wildly ramified:

$$
d^{2} \gamma_{r}\left(c_{2}\right)=u \gamma_{r-1}\left(c_{2}\right) \tau, \quad d^{2} c_{1}=0
$$

(iii) For A tamely ramified:

$$
d^{2} \pi \gamma_{r}\left(c_{2}\right)=\pi u \gamma_{r-1}\left(c_{2}\right) \tau, \quad d^{2}\left(c_{1} \gamma_{r}\left(c_{2}\right)\right)=u c_{1} \gamma_{r-1}\left(c_{2}\right) \tau
$$

and $d^{2}$ commutes with multiplication by $\pi$.

Proof. First we consider $A=\mathbf{Z}_{p}$, where

$$
E^{2}\left(\mathbf{Z}_{p}, \mathbf{Z} / p\right)=S_{\mathbf{F}_{p}}\{\sigma\} \otimes E_{\mathbf{F}_{p}}\{\tau\} .
$$

The spectral sequence converges to $\mathrm{THH}_{*}\left(\mathbf{Z}_{p}, \mathbf{Z} / p\right) \cong \pi_{*}\left(\mathrm{THH}\left(\mathbf{Z}_{p}\right) ; \mathbf{Z} / p\right)$ by (1.5). These groups were determined in [B1] by different methods, but we need only to know that $\mathrm{THH}_{1}\left(\mathbf{Z}_{p}, \mathbf{Z} / p\right)=0$, and this follows from general results: $\mathrm{THH}_{*}(R)=$ $K_{*}^{S}(R)$, the stable $K$-theory, by $\left[\mathrm{DM}\right.$, and $K_{*}^{S}(R)=\mathrm{HH}_{1}(R)$ by $\mathrm{K}$ for any ring $R$.

Since $\mathrm{THH}_{1}\left(\mathbf{Z}_{p}, \mathbf{Z} / p\right)=0$, it follows that $d^{2}(\sigma) \neq 0$, and we may choose $\sigma$ so that $d^{2}(\sigma)=\tau$. We have

$$
E^{2}(A \rightarrow A / p)=E^{2}\left(\mathbf{Z}_{p} \rightarrow \mathbf{Z} / p\right) \otimes \mathrm{HH}_{*}(A / p) .
$$

Since the spectral sequence is multiplicative, $d^{2}$ commutes with multiplication by $\pi$, so

$$
d^{2}\left(\sigma^{n}\right)=n \sigma^{n-1} \tau
$$

determines all differentials on $\pi^{i} \sigma^{n}$.

The differentials in the cases (ii) and (iii) follow from Proposition 4.2 and diagram (4.13).

Theorem 4.4. With the notation of Proposition 4.1, there are ring isomorphisms

(i) $\mathrm{THH}_{*}(A, A / p) \cong S_{k}\left\{\sigma^{p}\right\} \otimes_{k} E_{k}\left\{\sigma^{p-1} \tau\right\}, \quad A$ unramified,

(ii) $\mathrm{THH}_{*}(A, A / p) \cong S_{A / p}\left\{\alpha_{2}\right\} \otimes_{A} E_{A / p}\left\{\alpha_{1}\right\}, \quad A$ wildly ramified,

with $\operatorname{deg} \alpha_{1}=1$ and $\operatorname{deg} \alpha_{2}=2$, and $S_{R}\{-\}, E_{R}\{-\}$ denoting the symmetric and, respectively, exterior algebras of the listed elements over the ring $R$. 
Proof. Part (i) is obvious from formula (4.4) and propositions 4.1 and4.3. To prove (ii), we first observe that

$$
d^{2}: E_{2 n, 0}^{2} \rightarrow E_{2 n-2,1}^{2}
$$

is surjective. Indeed, if $r$ is the remainder of $i$ on division by $p$, i.e., $0 \leq r<p$ and $i \equiv r(\bmod p)$, then it follows from Proposition 4.1 (i), (ii) that

$$
d^{2}\left(\sum_{j=0}^{r}(-1)^{j} i^{[j]} u^{-j-1} \sigma^{i-j} \gamma_{n-i+j}\left(c_{2}\right)\right)=\sigma^{i} \gamma_{n-i-1}\left(c_{2}\right) \tau,
$$

where $i^{[j]}=i(i-1) \cdots(i-j+1)$, with the convention that $i^{[0]}=1$. The calculation uses the facts that $d^{2}$ is a derivation and that $i^{[r+1]} \equiv 0(\bmod p)$. We can multiply by $c_{1}$ to hit $\sigma^{i} \gamma_{n-i-1}\left(c_{2}\right) c_{1} \tau$ as well, so $d^{2}: E_{2 n+1,0}^{2} \rightarrow E_{2 n-1,1}^{2}$ is also surjective, and we get that $E_{*, 1}^{3}=0$.

The classes $\alpha_{2}=\sigma-u^{-1} c_{2}$ and $\alpha_{1}=c_{1}$ are $d^{2}$ cycles. Thus so are $\pi^{i} \alpha_{2}^{n}$ and $\pi^{i} \alpha_{1} \alpha_{2}^{n}$, and

$$
S_{k}\left\{\alpha_{2}\right\} \otimes_{k} E_{k}\left\{\alpha_{1}\right\} \subseteq E_{*, 0}^{3} .
$$

The opposite inclusion follows by counting dimensions.

Remark 4.5. Note as a special case of Theorem $4.4(\mathrm{i})$ that

$$
\mathrm{THH}_{*}\left(\mathbf{Z}_{p}, \mathbf{Z} / p\right) \cong S_{\mathbf{F}_{p}}\left\{f_{2 p}\right\} \otimes E_{\mathbf{F}_{p}}\left\{\varphi_{2 p-1}\right\}
$$

with $f_{2 p}=\sigma^{p}$ and $\varphi_{2 p-1}=\tau \sigma^{p-1}$. This result is due to Bökstedt, [B1]. Note further in case (ii) that the natural map

$$
i_{*}: \mathrm{THH}_{*}\left(\mathbf{Z}_{p}, \mathbf{Z} / p\right) \rightarrow \mathrm{THH}_{*}(A, A / p)
$$

associated with the inclusion $\mathbf{Z}_{p} \subset A$ maps $f_{2 p}$ to $\alpha_{2}^{p}$ and $\varphi_{2 p-1}$ to 0 .

Theorem 4.6. For tamely ramified $A$ there is an additive isomorphism

$$
\mathrm{THH}_{*}(A, A / p)=S_{k}\left\{\sigma^{p}\right\} \otimes_{k} E_{k}\left\{\sigma^{p-1} \tau\right\} \oplus \pi S_{A / p}\left\{\alpha_{2}\right\} \oplus \alpha_{1} S_{A / \pi^{e-1}}\left\{\alpha_{2}\right\} .
$$

The algebra structure of $\operatorname{THH}_{*}(A, A / p)$ for tamely ramified $A$ will be described in Proposition 5.6

Proof. Let

$$
i^{*}: \mathrm{THH}_{*}(A, A / p) \rightarrow \mathrm{THH}_{*}\left(A_{0}, A_{0} / p\right)
$$

be the map induced from the ring homomorphism

$$
A \rightarrow \operatorname{End}_{A_{0}}(A), \quad a \mapsto \text { multiplication by } a
$$

and Morita equivalence. It is split surjective in the tamely ramified case, split by a unit times the map $i_{*}$ induced by the inclusion $A_{0} \hookrightarrow A$ (as in remark 4.5): the composition $i^{*} i_{*}$ is induced by the following chain of maps:

$$
\begin{aligned}
& A_{0} / p\left(S^{x_{0}}\right) \otimes \mathbf{Z} A_{0} / p\left(S^{x_{1}}\right) \otimes \ldots \otimes \mathbf{Z} A_{0} / p\left(S^{x_{k}}\right) \\
& \stackrel{a \mapsto a \text {-id }}{\longrightarrow} \quad M_{e, e}\left(A_{0} / p\right)\left(S^{x_{0}}\right) \otimes \mathbf{Z} M_{e, e}\left(A_{0} / p\right)\left(S^{x_{1}}\right) \otimes \ldots \otimes \mathbf{Z} M_{e, e}\left(A_{0} / p\right)\left(S^{x_{k}}\right)
\end{aligned}
$$

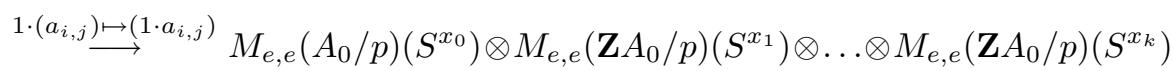

$$
\begin{aligned}
& \longrightarrow \quad M_{e, e}\left(A_{0} / p\left(S^{x_{0}}\right) \otimes \mathbf{Z} A_{0} / p\left(S^{x_{1}}\right) \otimes \ldots \otimes \mathbf{Z} A_{0} / p\left(S^{x_{k}}\right)\right) \\
& \stackrel{\operatorname{tr}}{\longrightarrow} \quad A_{0} / p\left(S^{x_{0}}\right) \otimes \mathbf{Z} A_{0} / p\left(S^{x_{1}}\right) \otimes \ldots \otimes \mathbf{Z} A_{0} / p\left(S^{x_{k}}\right),
\end{aligned}
$$


where $M_{e, e}(R)$ denotes $e$ by $e$ matrices over $R$ and the map before last is matrix multiplication. This composition is multiplication by $e$, the rank of $A$ over $A_{0}$, which we assume to be coprime to $p$.

The rest of the proof is similar to the proof of Theorem 4.4. First,

$$
\begin{aligned}
d^{2}\left(\sum_{j=0}^{r}(-1)^{j} i^{[j]} u^{-j-1} \sigma^{i-j} \pi^{a} \gamma_{n-i+j}\left(c_{2}\right)\right) & =\sigma^{i} \pi^{a} \gamma_{n-i-1}\left(c_{2}\right) \tau, \\
d^{2}\left(\sum_{j=0}^{r}(-1)^{j} i^{[j]} u^{-j-1} \sigma^{i-j} \pi^{a} \gamma_{n-i+j}\left(c_{2}\right) c_{1}\right) & =\sigma^{i} \pi^{a} \gamma_{n-i-1}\left(c_{2}\right) c_{1} \tau,
\end{aligned}
$$

with $a=1, \ldots, e-1$ in the first formula and $a=0, \ldots, e-2$ in the second, and with $r$ the remainder of $i$ on division by $p$. Since $\sigma^{n-1} \tau=d^{2}\left(\sigma^{n} / n\right)$ for $n \not \equiv 0(\bmod$ $p$ ), it follows that

$$
E_{*, 1}^{3}=k\left\langle\sigma^{p i-1} \tau \mid i=1,2, \ldots\right\rangle,
$$

the $k$-vector space spanned by the elements $\sigma^{p i-1} \tau$.

The kernel of $d^{2}: E_{*, 0}^{2} \rightarrow E_{*, 1}^{2}$ is the vector space spanned by the elements

$$
\begin{array}{ll}
\text { (a) } \pi^{j}\left(\sigma-u^{-1} c_{2}\right)^{i} c_{1}, & i \geq 0, j=0, \ldots, e-2, \\
\text { (b) } \pi^{j} \sigma^{p i}, & i \geq 0, j=0, \ldots, e-1, \\
\text { (c) } \pi^{j}\left(\sigma-u^{-1} c_{2}\right)^{i}, & i \not \equiv 0(\bmod p), j=1, \ldots, e-1 .
\end{array}
$$

In (a) and (c), the $i$ 'th power is to be interpreted as

$$
\left(\sigma-u^{-1} c_{2}\right)^{i}=\sum_{\nu=0}^{i}(-1)^{\nu} i^{[\nu]} \sigma^{i-\nu} u^{-\nu} \gamma_{\nu}\left(c_{2}\right) .
$$

(Note that $\pi^{j} c_{1} \gamma_{\nu}\left(c_{2}\right)$ for $j=0, \ldots, e-2$, and $\pi^{j} \gamma_{\nu}\left(c_{2}\right), j=1, \ldots, e-1$, are classes in $E_{*, 0}^{2}$.) This completes the argument.

\section{The Integral Result}

This section contains the proof of Theorem 1.1 We first reduce to the local situation. Let $B=\mathcal{O}(L)$, where $L$ is a finite extension of $\mathbf{Q}$. In positive degrees $\mathrm{THH}_{*}(B)$ consists of torsion groups. Indeed,

$$
\mathrm{THH}_{*}(B) \otimes \mathbf{Q} \cong \mathrm{HH}_{*}(B) \otimes \mathbf{Q} \cong \mathrm{HH}_{*}(L)=0 \text { for } *>0
$$

The first isomorphism reflects the fact that rationally $H(B)^{\wedge k}$ is homotopy equivalent to $H\left(B^{\otimes k}\right)$. The second isomorphism is obvious from the definition of Hochschild homology, and the vanishing of $\mathrm{HH}_{*}(L)$ is immediate from (4.6) because $L / \mathbf{Q}$ is separable.

By formula (5.1) it suffices to evaluate the $p$-adic homology groups of $\mathrm{THH}(B)$ for all primes $p$ or, what amounts to the same thing, the homotopy groups of the $p$-adic completion. Let us introduce the notation

$$
\mathrm{THH}_{*}^{\mathbf{Z}_{p}}(B)=\pi_{*}\left(\operatorname{THH}(B)_{p}^{\wedge}\right)=\pi_{*}\left(\operatorname{THH}(B) ; \mathbf{Z}_{p}\right)
$$

for the homotopy groups of the $p$-adic completion. By [HM], Addendum 6.2,

$$
\mathrm{THH}_{*}^{\mathbf{Z}_{p}}(B)=\mathrm{THH}_{*}^{\mathbf{Z}_{p}}\left(B \otimes \mathbf{Z}_{p}\right)
$$

when $B$ is finite over $\mathbf{Z}$. Similarly, for the linear Hochschild homology space

$$
\mathrm{HH}(B)_{p}^{\wedge} \simeq \mathrm{HH}\left(B \otimes \mathbf{Z}_{p}\right)_{p}^{\wedge} \simeq \mathrm{HH}^{\mathbf{Z}_{p}}\left(B \otimes \mathbf{Z}_{p}\right),
$$


where $\mathrm{HH}^{\mathbf{Z}_{p}}(-)$ denotes the Hochschild homology space over the ground ring $\mathbf{Z}_{p}$. Now,

$$
B \otimes \mathbf{Z}_{p}=\prod_{\wp \mid p} B_{\wp}, \quad \mathcal{D}_{B} \otimes \mathbf{Z}_{p}=\prod_{\wp \mid p} \mathcal{D}_{B_{\wp} / \mathbf{Z}_{p}}
$$

by $[\mathbf{S}$, Chap. III, proposition 10. Since THH of a product of rings is the product of THH of the factors, Theorem 1.1 is true in the global case if it is true locally: Theorem 1.1 follows from

Theorem 5.1. Let $A$ be a complete discrete evaluation ring of characteristic zero and with finite residue field $k$ of characteristic $p$. Then

$$
\begin{aligned}
\mathrm{THH}_{2 n-1}^{\mathbf{Z}_{p}}(A) & \cong \mathcal{D}_{A}^{-1} / n A, \\
\mathrm{THH}_{2 n}^{\mathbf{Z}_{p}}(A) & \cong 0 \text { for } n>0,
\end{aligned}
$$

where $\mathcal{D}_{A}^{-1}$ denotes the inverse different of $A / \mathbf{Z}$.

The rest of this section is devoted to a proof of Theorem 5.1 and $A$ will throughout be assumed to satisfy the conditions of the theorem.

The Witt vectors $A_{0}=W(k)$ form a subring of $A$, and

$$
\mathcal{D}_{A}=\mathcal{D}_{A / \mathbf{z}_{p}}=\mathcal{D}_{A / A_{0}},
$$

since $\mathcal{D}_{A / \mathbf{z}_{p}}=\mathcal{D}_{A / A_{0}} \mathcal{D}_{A_{0} / \mathbf{z}_{p}}$ and $\mathcal{D}_{A_{0} / \mathbf{z}_{p}}=A_{0}$. Let $e$ be the total ramification index of $A$. Then we have the values

$$
\begin{aligned}
\text { (i) } & \mathcal{D}_{A}=A \text { if } e=1, \\
\text { (ii) } & \mathcal{D}_{A}=\left(\pi^{m}\right)(m=e s+r, s \geq 1 \text { and } 0 \leq r<e) \text { if } p \mid e, \\
\text { (iii) } & \mathcal{D}_{A}=\left(\pi^{e-1}\right) \text { if }(p, e)=1,
\end{aligned}
$$

where $\pi \in A$ is a uniformizer, $c f$. [S], chap. III, theorem 1 and proposition 13 . We may write $A$ as

$$
A=A_{0}[\pi] /(\phi(\pi)),
$$

where $\phi$ is a degree $e$ Eisenstein polynomial. In this notation the different $\mathcal{D}_{A}=$ $\left(\phi^{\prime}(\pi)\right)$, where $\phi^{\prime}$ denotes the derivative of $\phi$, so

$$
\phi^{\prime}(\pi)=\epsilon \pi^{m}, \quad \text { resp. } \quad \phi^{\prime}(\pi)=\epsilon \pi^{e-1}, \quad \epsilon \in A^{\times},
$$

in cases (ii) and (iii) of (5.3). Recall also the unit $u \in A^{\times}$from (4.1), $\pi^{e}=p u$.

Theorem 5.1 is derived from the results of section 4 by a study of the Bockstein spectral sequence of $\operatorname{THH}(A)_{p}^{\wedge}$ (and $\left.\operatorname{HH}(A)_{p}^{\wedge}\right)$. This is the spectral sequence of the exact couple:

$$
\begin{array}{ccc}
\pi_{*}\left(\operatorname{THH}(A)_{p}^{\wedge}\right) & \stackrel{p}{\longrightarrow} & \pi_{*}\left(\operatorname{THH}(A)_{p}^{\wedge}\right) \\
& \pi_{*}\left(\operatorname{THH}(A)_{p}^{\wedge} ; \mathbf{Z} / p\right) & \swarrow
\end{array}
$$

In view of the discussion leading to (1.5), or (3.7),

$$
\pi_{*}\left(\operatorname{THH}(A) ; \mathbf{Z} / p^{i}\right) \cong \operatorname{THH}_{*}\left(A, A / p^{i}\right),
$$

so

$$
\begin{aligned}
E^{1}(A) & =\operatorname{THH}_{*}(A, A / p), \\
E^{\infty}(A) & =\left(\operatorname{THH}(A)_{p}^{\wedge} / \text { torsion }\right) \otimes \mathbf{Z} / p,
\end{aligned}
$$


and similarly for $\operatorname{HH}(A)_{p}^{\wedge}$. The higher differentials are induced from the additive relations

$$
\partial_{r}: \operatorname{THH}_{*}(A, A / p) \stackrel{\rho_{r}}{\longleftarrow} \mathrm{THH}_{*}\left(A, A / p^{r}\right) \stackrel{\beta_{r}}{\longrightarrow} \mathrm{THH}_{*}(A) \stackrel{\rho}{\longrightarrow} \mathrm{THH}_{*}(A, A / p),
$$

where $\beta_{r}$ is the connecting homomorphism in the analogue of (5.6) with $p$ replaced by $p^{r}$. Elements in the image of $\partial_{r}$ represent torsion of order $p^{r}$. In our case

$$
E_{*}^{\infty}\left(\operatorname{THH}(A)_{p}^{\wedge}\right)=0 \text { for } *>0
$$

by the argument of (5.1). We refer the reader to $\mathrm{Bro}$ for questions relating to the Bockstein spectral sequence, and note that as $\operatorname{THH}(A)$ is a simplicial abelian group the homotopy groups above can all be viewed as homology groups of the induced chain complexes, and $\beta_{r}=\frac{1}{p^{r}} d$.

We begin with the Bockstein spectral sequence for $\operatorname{HH}(A)_{p}^{\wedge}$. The $E^{1}$-term,

$$
E_{*}^{1}\left(\mathrm{HH}(A)_{p}^{\wedge}\right)=\operatorname{HH}_{*}(A, A / p)=\operatorname{HH}_{*}(A / p),
$$

was listed in Proposition 4.1. If $e=1$ everything collapses to degree zero, and there is nothing to prove. If $e>1$ we have, with the notation from Proposition 4.1 and (5.3),

Lemma 5.2. In the Bockstein spectral sequence for $\operatorname{HH}(A)_{p}^{\wedge}$ we have:

(i) If $p$ divides $e$, then

$$
\begin{aligned}
\partial_{s}\left(\pi^{i} \gamma_{k}\left(c_{2}\right)\right) & =\left(\epsilon u^{s}\right) \pi^{i+r} c_{1} \gamma_{k-1}\left(c_{2}\right) \quad \text { if } i+r<e, \\
\partial_{s+1}\left(\pi^{i} \gamma_{k}\left(c_{2}\right)\right) & =\left(\epsilon u^{s+1}\right) \pi^{i+r-e} c_{1} \gamma_{k-1}\left(c_{2}\right) \quad \text { if } i+r \geq e .
\end{aligned}
$$

(ii) If $(p, e)=1$, then

$$
\partial_{1}\left(\pi^{i} \gamma_{k}\left(c_{2}\right)\right)=(\epsilon u) \pi^{i-1} c_{1} \gamma_{k-1}\left(c_{2}\right) .
$$

Proof. The units $\left(\epsilon u^{s}\right)$, etc., are of course quite immaterial, but we keep track of them anyway. First, recall that the natural map

$$
\mathrm{HH}_{*}^{\mathbf{Z}_{p}}\left(A, A / p^{i}\right) \rightarrow \mathrm{HH}_{*}^{A_{0}}\left(A, A / p^{i}\right)
$$

is an isomorphism by a change of ring argument (cf. the proof of Proposition 4.1), so we use $A_{0}$ as ground ring, and can use the chain complexes

$$
0 \rightarrow Z_{*}^{A_{0}}(A, A) \stackrel{p^{i}}{\rightarrow} Z_{*}^{A_{0}}(A, A) \rightarrow Z_{*}^{A_{0}}\left(A, A / p^{i}\right) \rightarrow 0,
$$

cf. (4.6), (4.8), to calculate

$$
\beta_{i}: \mathrm{HH}_{*}^{A_{0}}\left(A, A / p^{i}\right) \rightarrow \mathrm{HH}_{*}^{A_{0}}(A, A) .
$$

In case (i), $\gamma_{k}\left(c_{2}\right)$ and $c_{1} \gamma_{k-1}\left(c_{2}\right)$ are the generators of $Z_{2 k}^{A_{0}}\left(A, A / p^{s}\right)$ and $Z_{2 k-1}^{A_{0}}(A, A)$ respectively, and

$$
\beta_{s}\left(\gamma_{r}\left(c_{2}\right)\right)=\left(\phi^{\prime}(\pi) / p^{s}\right) c_{1} \gamma_{r-1}\left(c_{2}\right)=\left(\epsilon u^{s}\right) c_{1} \gamma_{r-1}\left(c_{2}\right) .
$$

Now (i) follows easily since $\pi^{e}=p u$. Case (ii) is completely similar.

Our calculation below of the higher differentials $\partial_{s}$ in $E_{*}^{s}\left(\operatorname{THH}(A)_{p}^{\wedge}\right)$ is inspired by Bökstedt's calculations from [B1] in the case $A=\mathbf{Z}_{p}$. It uses some general structure theory for $\operatorname{THH}(A)$ when $A$ is commutative, which we briefly recall. The point is that $\operatorname{THH}(A)$ is the zero'th space of a so-called $H_{\infty}$-ring spectrum $\mathrm{TH}(A)$. 
The reader is referred to BMMS for the general theory of $H_{\infty}$-ring spectra. We shall just need to know that there is a spectrum map

$$
D_{n}: E \Sigma_{n} \ltimes_{\Sigma_{n}} \mathrm{TH}(A)^{\wedge n} \rightarrow \mathrm{TH}(A)
$$

that extends the $n$-fold product. The construction of $D_{n}$ was given initially in [B1], but it follows also from [HM], sect. 2.7, or most elegantly from the theory of $S$-modules developed in EKMM]. The map $D_{p}$ implies that the $\bmod p$ spectrum homology of $\operatorname{TH}(A)$ has mod $p$ homology (Dyer-Lashof) operations $Q^{i}$. For our purpose, let $\mathrm{THH}_{\sharp}(A)$ denote the chain complex of the original simplicial abelian group, and let $\mathrm{THH}_{\sharp}\left(A, A / p^{i}\right)$ be the chain complex with $\mathbf{Z} / p^{i}$ coefficients, whose homology is $\operatorname{THH}_{*}\left(A, A / p^{i}\right)$. Then $D_{p}$ induces a map

$$
P_{*} \otimes_{\mathbf{Z}_{p}\left[C_{p}\right]} \operatorname{THH}_{\sharp}\left(A, A / p^{i}\right)^{\otimes p} \rightarrow \mathrm{THH}_{\sharp}\left(A, A / p^{i}\right),
$$

where $P_{*}$ is the standard $\mathbf{Z}_{p}\left[C_{p}\right]$-free resolution of $\mathbf{Z}_{p}$. This defines homology operations and Pontrjagin powers (which refine the $p$ 'th power):

$$
\begin{aligned}
& Q^{i}: \operatorname{THH}_{*}(A, A / p) \rightarrow \mathrm{THH}_{*}(A, A / p), \\
& \mathcal{P}: \operatorname{THH}_{n}\left(A, A / p^{i}\right) \rightarrow \operatorname{THH}_{p n}\left(A, A / p^{i+1}\right) \text {. }
\end{aligned}
$$

The defining formulas and general properties can be found in [CLM], pages 5-15 and $62-66$, or for $p=2$ in [M1].

We shall need only very little of this structure, namely that the differentials $\partial_{s}$ in (5.6) are derivations, and the following formulas: for $x \in \operatorname{im}\left(\mathrm{THH}_{2 n}\left(A, A / p^{s}\right) \rightarrow\right.$ $\left.\mathrm{THH}_{2 n}(A, A / p)\right)$, i.e. for $x \in E^{1}$ which survives to $E^{s}$,

$$
\begin{aligned}
\partial_{s+1}\left(x^{p}\right) & =\partial_{s}(x) x^{p-1} \quad \text { if } p>2 \text { or } s>1, \\
\partial_{2}\left(x^{2}\right) & =x \partial_{1}(x)+Q^{2 n}\left(\partial_{1} x\right) .
\end{aligned}
$$

In short, (5.8) implies that the differential in $\operatorname{THH}_{\sharp}(A)$ is a derivation up to certain correction terms, such as the second term in the formula for $\partial_{2}$ (see e.g. [M1).

We now study the Bockstein spectral sequence for $\operatorname{THH}(A)_{p}^{\wedge}$, dividing up the calculations into the three cases of $(5.3)$.

Proposition 5.3 (M. Bökstedt). If $e=1$, then

$$
\operatorname{THH}_{*}(A, A / p)=S_{k}\left\{f_{2 p}\right\} \otimes_{k} E_{k}\left\{\varphi_{2 p-1}\right\}
$$

and, for $n=p^{t} n^{\prime}$ with $\left(n^{\prime}, p\right)=1$,

$$
\partial_{t+1}\left(f_{2 p}^{n}\right)=n^{\prime} \varphi_{2 p-1} f_{2 p}^{n-1} .
$$

Proof. The first part is Theorem 4.4(i) with

$$
f_{2 p}=\sigma^{p}, \varphi_{2 p-1}=\tau \sigma^{p-1} .
$$

These elements are in the image of $\mathrm{THH}_{*}\left(\mathbf{Z}_{p}, \mathbf{Z} / p\right) \rightarrow \mathrm{THH}_{*}(A, A / p)$, so the Bockstein formulas follow from [B1].

Remark 5.4. The paper [B1] has never appeared in print, so it is in order to remark that the calculation of Mac Lane cohomology in [FP] also gives the result, up to a unit (which is all we will need in the calculation to follow). Indeed, by Corollary 3.2 of [FP],

$$
\mathrm{HML}^{2 i}(\mathbf{Z}, \mathbf{Z})=\mathbf{Z} / i, \quad \mathrm{HML}^{2 i+1}(\mathbf{Z}, \mathbf{Z})=0 .
$$


On the other hand, $\mathrm{HML}_{*}(\mathbf{Z}, \mathbf{Z}) \cong \mathrm{THH}_{*}(\mathbf{Z})$ by $[\mathrm{PW}$, and the universal coefficient theorem becomes

$$
0 \rightarrow \operatorname{Ext}^{1}\left(\mathrm{THH}_{2 i-1}(\mathbf{Z}), \mathbf{Z}\right) \rightarrow \mathrm{HML}^{2 i}(\mathbf{Z}, \mathbf{Z}) \rightarrow \operatorname{Hom}\left(\mathrm{THH}_{2 i}(\mathbf{Z}), \mathbf{Z}\right) \rightarrow 0 .
$$

This recovers Bökstedt's result that

$$
\mathrm{THH}_{2 i-1}(\mathbf{Z})=\mathbf{Z} / i \text {. }
$$

For $i=p$ this implies that $\partial_{1}\left(f_{2 p}\right)=$ unit $\cdot \varphi_{2 p-1}$. The higher Bocksteins then follow from (5.9), except for the case when $p=2$ and $n=2$. In that case we must argue that

$$
\partial_{2}\left(f_{4}^{2}\right)=f_{4} \varphi_{3}+Q^{4}\left(\varphi_{3}\right) \neq 0 .
$$

But if $\partial_{2}\left(f_{4}^{2}\right)=0$, then $\partial_{s}\left(f_{4}^{2}\right) \neq 0$ for some $s>2\left(\right.$ since $\pi_{*}\left(\operatorname{THH}\left(\mathbf{Z}_{p}\right)\right) \otimes \mathbf{Q}=0$ for $*>0$ ). This would imply that $f_{4} \varphi_{3}$ was the mod 2 restriction of a class of order $2^{s}$, contradicting the fact that $\mathrm{THH}_{7}(\mathbf{Z})=\mathbf{Z} / 4$.

Proposition 5.5. If $p$ divides $e$, then

$$
\mathrm{THH}_{*}(A, A / p)=S_{A / p}\left\{\alpha_{2}\right\} \otimes_{A} E_{A / p}\left\{\alpha_{1}\right\} .
$$

If $n=p^{t} n^{\prime},\left(n^{\prime}, p\right)=1$, then, with the notation of (5.3),

$\begin{array}{ll}\text { (i) } \partial_{s+t}\left(\pi^{i} \alpha_{2}^{n}\right)=\text { unit } \cdot \pi^{i+r} \alpha_{1} \alpha_{2}^{n-1} & \text { if } i+r<e, \\ \text { (ii) } \partial_{s+t+1}\left(\pi^{i} \alpha_{2}^{n}\right)=\text { unit. } \pi^{i+r-e} \alpha_{1} \alpha_{2-1} & \text { if } i+r \geq e .\end{array}$

(ii) $\partial_{s+t+1}\left(\pi^{i} \alpha_{2}^{n}\right)=$ unit $\cdot \pi^{i+r-e} \alpha_{1} \alpha_{2}^{n-1}$ if $i+r \geq e$.

Proof. The first statement is Theorem4.4(ii). For the Bockstein structure, consider the commutative diagram

$$
\begin{array}{ccc}
\operatorname{THH}\left(A, A / p^{i}\right) & \stackrel{\text { red }}{\longrightarrow} & \operatorname{THH}\left(A / p^{i}, A / p^{i}\right) \\
\downarrow L & & \downarrow L \\
\operatorname{HH}\left(A, A / p^{i}\right) & \stackrel{\text { red }}{\longrightarrow} & \operatorname{HH}\left(A / p^{i}, A / p^{i}\right)
\end{array}
$$

where the reduction of the bottom row induces a homeomorphism. The element $\alpha_{2} \in \mathrm{THH}_{2}(A, A / p)$ maps to $\left(\sigma-u^{-1} c_{2}\right) \in \mathrm{THH}_{2}(A / p)$ and further by $L$ into $-u^{-1} c_{2} \in \mathrm{HH}_{2}(A / p)$, so the diagram gives

$$
L_{*}\left(\alpha_{2}\right)=-u^{-1} c_{2} \in \mathrm{HH}_{2}(A, A / p) .
$$

Since $L_{*}$ is an isomorphism in degree 1 , Lemma 5.2 yields

$$
\alpha_{2} \in \operatorname{im}\left(\mathrm{THH}_{2}\left(A, A / p^{s}\right) \rightarrow \mathrm{THH}_{2}(A, A / p)\right)
$$

and

$$
\partial_{s}\left(\alpha_{2}\right)=-\left(\epsilon u^{s-1}\right) \cdot \pi^{r} \alpha_{1} .
$$

Formula (5.9) then gives the stated value of $\partial_{s+t}\left(\alpha_{2}^{n}\right)$ except if $s=1$ and $p=2$. Once we know that

$$
\partial_{s+t}\left(\alpha_{2}^{n}\right)=\text { unit } \cdot \pi^{r} \alpha_{1} \alpha_{2}^{n-1},
$$

we know that there exists some chain $\widehat{\alpha_{2}^{n}} \in \operatorname{THH}_{\sharp}(A)$ whose reduction to $\mathrm{THH}_{\sharp}(A, A / p)$ represents $\alpha_{2}^{n}$ for which

$$
d\left(\widehat{\alpha_{2}^{n}}\right)=p^{s+t} \cdot \text { unit } \cdot \pi^{r} \widehat{\alpha_{1} \alpha_{2}^{n-1}}+p^{s+t+1} \widehat{x}
$$

where $\widehat{\alpha_{1} \alpha_{2}^{n-1}}$ is a chain whose reduction to $\operatorname{THH}_{\sharp}(A, A / p)$ represents $\alpha_{1} \alpha_{2}^{n-1}$, and $\widehat{x}$ is a chain whose reduction represents some class $x \in \mathrm{THH}_{2 n-1}(A, A / p)$. We use 
the $A$-module structure of $\mathrm{THH}_{\sharp}(A)$, and consider the chain $\pi^{i} \widehat{\alpha_{2}^{n}}$, whose reduction represents $\pi^{i} \alpha_{2}^{n}$ :

$$
d\left(\pi^{i} \widehat{\alpha_{2}^{n}}\right)=p^{s+t} \cdot \text { unit } \cdot \pi^{i+r} \widehat{\alpha_{1} \alpha_{2}^{n-1}}+p^{s+t+1} \pi^{i} \widehat{x} .
$$

For $i+r<e$, this shows that $\partial_{s+t}\left(\pi^{i} \alpha_{2}^{n}\right)=$ unit $\cdot \pi^{i+r} \alpha_{1} \alpha_{2}^{n-1}$, as desired. For $i+r \geq e$, we can rewrite (5.11) as

$$
d\left(\pi^{i} \widehat{\alpha_{2}^{n}}\right)=p^{s+t+1}\left(\text { unit } \cdot \pi^{i+r-e} \widehat{\alpha_{1} \alpha_{2}^{n-1}}+\pi^{i} \widehat{x}\right) .
$$

Since $x \in \mathrm{THH}_{2 n-1}(A, A / p)$, it follows that $x=a \cdot \alpha_{1} \alpha_{2}^{n-1}$ for some $a \in A / p$, so by (5.12) we can say that

$$
\partial_{s+t+1}\left(\left(\pi^{i} \alpha_{2}^{n}\right)=\left(\text { unit } \cdot \pi^{i+r-e}+\pi^{i} a\right) \alpha_{1} \alpha_{2}^{n-1}=\text { unit } \cdot \pi^{i+r-e} \alpha_{1} \alpha_{2}^{n-1},\right.
$$

where the second unit may be different than the first, but is nevertheless a unit because $r-e<0$. This completes the calculation, except for the special case of $p=2$ and $s=1$.

In the $p=2, s=1$ case, we know that $\partial_{1}$ vanishes on $\operatorname{THH}_{4}(A, A / 2)=A / 2 \cdot \alpha_{2}^{2}$, so we know that there are no direct summands of order 2 in $\mathrm{THH}_{3}(A)$. Formula (5.9) becomes

$$
\begin{aligned}
\partial_{2}\left(\alpha_{2}^{2}\right) & =-\epsilon \pi^{r} \alpha_{1} \alpha_{2}+Q^{2}\left(-\epsilon \pi^{r} \alpha_{1}\right) \\
& =-\epsilon \pi^{r} \alpha_{1} \alpha_{2}+Q^{2}\left(\epsilon \pi^{r} \alpha_{1}\right)=\zeta \alpha_{1} \alpha_{2},
\end{aligned}
$$

since $\mathrm{THH}_{3}(A, A / 2)=A / 2 \cdot \alpha_{1} \alpha_{2}$, and we need to show that $\zeta=$ unit $\cdot \pi^{r}$.

To show this, we calculate $\mathrm{THH}_{3}(A)$ directly, using the spectral sequence of $A$-modules with

$$
E_{a, b}^{2}=\operatorname{HH}_{a}\left(A, \mathrm{THH}_{b}(\mathbf{Z}) \otimes A\right)
$$

converging to $\mathrm{THH}_{*}(A)$ (due to Pirashvili and Waldhausen, in Theorem 4.1 of [PW]; other derivations can be found in Theorem 6.2.8 of [Brun1] or Corollary 3.3 of [L2]). The differentials of this spectral sequence are harder to analyze than those of the spectral sequence of Theorem 3.3. but it turns out that they do not affect the terms of total degree 3 .

We start with the special case $A=\mathbf{Z}$ of Theorem 1.1 done by Bökstedt, which can already be deduced from his Theorem 5.3 using the discussion in the beginning of this section. It tells us that $\mathrm{THH}_{0}(\mathbf{Z}) \cong \mathbf{Z}, \mathrm{THH}_{1}(\mathbf{Z}) \cong \mathrm{THH}_{2}(\mathbf{Z})=0$, and $\mathrm{THH}_{3}(\mathbf{Z}) \cong \mathbf{Z} / 2$. Therefore in the spectral sequence (5.14), $E_{*, 0}^{2} \cong \mathrm{HH}_{*}(A)$ as given in equation (4.9), $E_{*, 1}^{2} \cong E_{*, 2}^{2}=0$, and $E_{*, 3}^{2} \cong \operatorname{HH}_{*}(A / 2)$ as given in Proposition 4.1. No nonzero differentials can land or originate in the terms of total degree 3 , so we get a short exact sequence of $A$-modules

$$
0 \rightarrow \mathrm{HH}_{0}(A / 2) \rightarrow \mathrm{THH}_{3}(A) \rightarrow \mathrm{HH}_{3}(A) \rightarrow 0 .
$$

Recalling that $(2)=\left(\pi^{e}\right)$, this becomes

$$
0 \rightarrow A / \pi^{e} \rightarrow \mathrm{THH}_{3}(A) \rightarrow A / \pi^{m} \rightarrow 0 .
$$

Since $A$ is a discrete valuation ring,

$$
\mathrm{THH}_{3}(A) \cong \bigoplus_{i=1}^{q} A / \pi^{k_{i}} .
$$


Since $\mathrm{THH}_{3}(A)$ as an additive group contains no direct summands of order 2, all the $k_{i}$ must be greater than or equal to $2 e$. By counting elements in (5.15), we see that the sum of the $k_{i}$ must be $e+m=2 e+r$ with $r<e$, so $q=1$ and

$$
\mathrm{THH}_{3}(A) \cong A / \pi^{e+m}
$$

as an $A$-module. This means that, as an abelian group, it is isomorphic to

$$
\bigoplus_{i=1}^{(e-r) \cdot[k: \mathbf{Z} / 2]} \mathbf{Z} / 4 \oplus \bigoplus_{i=1}^{r \cdot[k: \mathbf{Z} / 2]} \mathbf{Z} / 8
$$

where $k=A_{0} / 2$.

Now we go back to analyzing $\zeta$ in (5.13). If

$$
\partial_{2}\left(\alpha_{2}^{2}\right)=\text { unit } \cdot \pi^{t} \alpha_{1} \alpha_{2},
$$

we get an $(e-t) \cdot[k: \mathbf{Z} / 2]$-dimensional subspace of $\operatorname{THH}_{4}(A, A / 2)$ where $\partial_{2}$ does not vanish, and a $t \cdot[k: \mathbf{Z} / 2]$-dimensional subspace where it does. So we get that as an abelian group, $\mathrm{THH}_{3}(A)$ contains $\bigoplus_{i=1}^{(e-t) \cdot[k: \mathbf{Z} / 2]} \mathbf{Z} / 4$ as a direct summand, and the rest of it consists of cyclic groups of order 8 and above. Comparing this with our actual calculation of $\mathrm{THH}_{3}(A)$, we deduce that $t=r$.

Proposition 5.6. Suppose $(e, p)=1$. Then

(i) We have

$\mathrm{THH}_{*}(A, A / p)=\mathrm{THH}_{*}\left(A_{0}, A_{0} / p\right) \oplus \pi S_{A / p}\left\{\alpha_{2}\right\} \oplus \alpha_{1} S_{A / \pi^{e-1}}\left\{\alpha_{2}\right\}$,

where $\mathrm{THH}_{*}\left(A_{0}, A_{0} / p\right)=S_{k}\left\{f_{2 p}\right\} \otimes_{k} E_{k}\left\{\varphi_{2 p-1}\right\}$.

(ii) There are the relations

$$
\pi f_{2 p}^{n}=\pi \alpha_{2}^{n p}, \quad \pi^{e-1} \alpha_{1} \alpha_{2}^{p n-1}=-\left(u \epsilon^{-1}\right) \varphi_{2 p-1} f_{2 p}^{n-1} .
$$

(iii) For $n=p^{t} n^{\prime},\left(n^{\prime}, p\right)=1$,

$$
\begin{aligned}
\partial_{1+t}\left(\pi^{i} \alpha_{2}^{n}\right) & =-\left(\epsilon n^{\prime}\right) \pi^{i-1} \alpha_{1} \alpha_{2}^{n-1}, \quad 0<i<e, \\
\partial_{1+t}\left(f_{2 p}^{n}\right) & =n^{\prime} \varphi_{2 p-1} f_{2 p}^{n-1} .
\end{aligned}
$$

Proof. Part (i) is Theorem 4.4(i) and Theorem 4.6. The first relation of (ii) is by definition of $\alpha_{2}$ and $f_{2 p}$. The second formula of (iii) is Proposition 5.3. We prove the first formula of (iii) and then derive the second formula of (ii). It suffices to calculate $\partial_{1+t}\left(\pi \alpha_{2}^{n}\right)$, since $\partial_{1+t}$ commutes with multiplication by scalars such as $\pi^{i-1}$.

If $t=0$, then $\partial_{1}\left(\pi \alpha_{2}^{n}\right)$ can be found using Lemma 5.2 (ii) because $\partial_{1}$ is a derivation. So suppose $t \geq 1$ and write $n=p m$. Then $\pi \alpha_{2}^{n}=\pi f_{2 p}^{m}$ and $\partial_{t}\left(f_{2 p}^{m}\right)=$ $n^{\prime} \varphi_{2 p-1} f_{2 p}^{m-1}$. We apply

$$
\operatorname{THH}\left(A_{0}, A_{0} / p\right) \stackrel{\pi \cdot}{\longrightarrow} \operatorname{THH}(A, A / p)
$$

to get information on $\partial_{1+t}\left(\pi f_{2 p}^{m}\right)$. In the spectral sequence

$$
\mathrm{THH}_{*}\left(A / p, \operatorname{Tor}_{*}^{A}(A / p, A / p)\right) \Rightarrow \mathrm{THH}_{*}(A, A / p)
$$


$\pi \varphi_{2 p-1} f_{2 p}^{m-1}$ corresponds to the class $\pi \tau \sigma^{n-1}$, which is hit by a $d^{2}$-differential, namely

$$
\begin{aligned}
d^{2}\left(x_{2 n}\right) & =\pi \tau \sigma^{n-1}, \\
x_{2 n} & =\sum_{j=0}^{n-1}(-1)^{j}(n-1)^{[j]} u^{-j-1} \sigma^{n-1-j} \pi \gamma_{j+1}\left(c_{2}\right) .
\end{aligned}
$$

Therefore, $\partial_{t}\left(\pi f_{2 p}^{m}\right)=0$, and $\pi f_{2 p}^{m}$ lifts to an element of $\operatorname{THH}_{2 n}\left(A, A / p^{t}\right)$, whose image under $\partial_{1+t}$ we must calculate.

Since $\operatorname{THH}(A, M)$ is a simplicial abelian group, its homotopy groups can be viewed as homology groups of the induced chain complex. The relation (5.16) yields a chain $\hat{x}_{2 n}$ of $\operatorname{THH}(A)$ with

$$
d \hat{x}_{2 n}=\pi \varphi_{2 p-1} f_{2 p}^{m-1}+p \hat{y}_{2 n-1} .
$$

On the chain level,

$$
d\left(\pi f_{2 p}^{m}-n \hat{x}_{2 n}\right)=-p n \hat{y}_{2 n-1}
$$

and hence

$$
\partial_{1+t}\left(\pi f_{2 p}^{m}\right)=-n^{\prime} \partial_{1}\left(x_{2 n}\right)
$$

We use Lemma 5.2 (ii) to calculate the right hand side:

$$
\begin{aligned}
\partial_{1}\left(x_{2 n}\right) & =\epsilon \sum_{j=0}^{n-1}(-1)^{j}(n-1)^{[j]} u^{-j} \sigma^{n-1-j} c_{1} \gamma_{j}\left(c_{2}\right) \\
& =\epsilon \sum_{j=0}^{n-1}(-1)^{j}\left(\begin{array}{c}
n-1 \\
j
\end{array}\right) u^{-j} \sigma^{n-1-j} c_{1} c_{2}^{j} \\
& =\epsilon\left(\sigma-u^{-1} c_{2}\right)^{n-1} c_{1}=\epsilon \alpha_{1} \alpha_{2}^{n-1} .
\end{aligned}
$$

Finally, the second relation in (ii) is a consequence of (5.17) upon multiplication by $\pi^{e-1}$. Indeed, on the one hand

$$
\begin{aligned}
\partial_{1+t}\left(\pi^{e} f_{2 p}^{m}\right) & =\partial_{1+t}\left(p u f_{2 p}^{m}\right) \\
& =u \partial_{t}\left(f_{2 p}^{m}\right) \\
& =\left(u n^{\prime}\right) \varphi_{2 p-1} f_{2 p}^{m-1}
\end{aligned}
$$

and on the other hand,

$$
\pi^{e-1} \partial_{1+t}\left(\pi f_{2 p}^{m}\right)=-\left(n^{\prime}\right) \pi^{e-1} \epsilon \alpha_{1} \alpha_{2}^{n-1}
$$

by the above calculation of $\partial_{1}\left(x_{2 n}\right)$.

Proof of Theorem [5.1. The exact sequences

$$
\cdots \longrightarrow \mathrm{THH}_{*}^{\mathbf{Z}_{p}}\left(A, A / p^{s}\right) \stackrel{\beta_{s}}{\longrightarrow} \mathrm{THH}_{*}^{\mathbf{Z}_{p}}(A) \stackrel{p^{s}}{\longrightarrow} \mathrm{THH}_{*}^{\mathbf{Z}_{p}}(A) \longrightarrow \cdots
$$

and the commutative diagram

$$
\begin{array}{ccc}
\mathrm{THH}_{*}^{\mathbf{Z}_{p}}\left(A, A / p^{s-1}\right) & \stackrel{p_{*}}{\longrightarrow} & \mathrm{THH}_{*}^{\mathbf{Z}_{p}}\left(A, A / p^{s}\right) \\
\searrow \beta_{s-1} & \mathrm{THH}_{*}^{\mathbf{Z}_{p}}(A) & \swarrow \beta_{s}
\end{array}
$$

show that the elements in the image of the additive relation $\partial_{s}$ are mod $p$ reductions of elements in $\mathrm{THH}_{*}^{\mathbf{Z}_{p}}(A)$ of order $p^{s}$. Hence Propositions [5.3, 5.5 and [5.6] give 
the additive structure of $\mathrm{THH}_{*}^{\mathbf{Z}_{p}}(A)$. In positive dimensions there are no even dimensional classes $\left(E_{2 n}^{\infty}=0\right.$ for $\left.n>0\right)$.

The non-zero groups in odd dimension can be listed as follows. Let $t=\nu_{p}(n)$ be the $p$-adic valuation of $n$ and let $A_{0}=W(k)$ be the unramified part of $A$.

If $e=1$, we have $A=A_{0}=\mathcal{D}_{A}^{-1}$ and

$$
\mathrm{THH}_{2 n-1}^{\mathbf{Z}_{p}}(A)=A_{0} / p^{t} A_{0}\left\langle g_{2 n-1}\right\rangle .
$$

The mod $p$ reduction of $g_{2 n-1}$ is $\rho_{\mathbf{Z} / p}\left(g_{2 n-1}\right)=\varphi_{2 p-1} f_{2 p}^{(n / p)-1}$.

For $(e, p)>1$ we have $\mathcal{D}_{A}^{-1}=\left(\pi^{-m}\right)$ with $m=s e+r, s \geq 1$ and $0 \leq r<e$, and also

$$
\mathrm{THH}_{2 n-1}^{\mathbf{Z}_{p}}(A)=\bigoplus_{i=0}^{r-1} A_{0} / p^{s+t+1} A_{0}\left\langle a_{2 n-1, i}\right\rangle \oplus \bigoplus_{i=r}^{e-1} A_{0} / p^{s+t} A_{0}\left\langle a_{2 n-1, i}\right\rangle .
$$

The mod $p$ reductions of the generators are

$$
\rho_{\mathbf{Z} / p}\left(a_{2 n-1}, i\right)=\pi^{i} \alpha_{1} \alpha_{2}^{n-1}= \begin{cases}\partial_{s+t+1}\left(\pi^{i+e-r} \alpha_{2}^{n}\right), & 0 \leq i<r \\ \partial_{s+t}\left(\pi^{i-r} \alpha_{2}^{n}\right), & r \leq i<e\end{cases}
$$

If $t>0$, since $\alpha_{2}^{n}=f_{2 p}^{n / p}$ and $\beta_{t}\left(f_{2 p}^{n / p}\right)=g_{2 n-1}$, the map

$$
i_{*}: \mathrm{THH}_{*}^{\mathbf{Z}_{p}}\left(A_{0}\right) \rightarrow \mathrm{THH}_{*}^{\mathbf{Z}_{p}}(A),
$$

induced from the inclusion, maps $g_{2 n-1}$ into $p^{s} a_{2 n-1, r}=p^{s} \pi^{r} a_{2 n-1,0}$, so formally $\pi^{-m} g_{2 n-1}=a_{2 n-1,0}$, and

$$
\mathrm{THH}_{2 n-1}^{\mathbf{Z}_{p}}(A) \cong \mathcal{D}_{A}^{-1} / n A
$$

with $1(\bmod n A)$ corresponding to $g_{2 n-1}$.

For $(e, p)=1$ we have $\mathcal{D}_{A}^{-1}=\left(\pi^{1-e}\right)$ and

$$
\mathrm{THH}_{2 n-1}^{\mathbf{Z}_{p}}(A)=\bigoplus_{i=0}^{e-2} A_{0} / p^{t+1} A_{0}\left\langle a_{2 n-1, i}\right\rangle \oplus A_{0} / p^{t} A_{0}\left\langle g_{2 n-1}\right\rangle
$$

with $\rho_{\mathbf{Z} / p}\left(a_{2 n-1, i}\right)=\pi^{i} \alpha_{1} \alpha_{2}^{n-1}$, and for $t>0$

$$
\rho_{\mathbf{Z} / p}\left(\pi^{e-1} a_{2 n-1, i}\right)=\rho_{\mathbf{Z} / p}\left(g_{2 n-1}\right) .
$$

Again we formally have $\pi^{1-e} g_{2 n-1}=a_{2 n-1,0}$.

Remark 5.7. In [FP] Franjou and Pirashvili calculated the so-called Mac Lane cohomology of $\mathbf{Z}$, from which one can derive $\mathrm{THH}_{*}(\mathbf{Z})$. One might expect that one could similarly calculate Mac Lane cohomology of the rings in Theorem 1.1, using the spectral sequence of Pirashvili, dual to 3.3. The Mac Lane cohomology groups are Ext-groups in the functor category $\mathcal{F}(R)$, and therefore, in the case that $R$ is a $\mathrm{PID}$, associated to $\mathrm{THH}_{*}(R)$ by the usual short exact sequence. Thus, the expected value for the rings $A$ of Theorem 1.1 is

$$
\mathrm{HML}^{2 n}(A)=\mathcal{D}_{A}^{-1} / n A .
$$




\section{REFERENCES}

[B] M. Bökstedt, Topological Hochschild homology, preprint (Bielefeld).

[B1] M. Bökstedt, Topological Hochschild homology of $\mathbf{Z}$ and $\mathbf{Z} / p$, preprint (Bielefeld).

[BM] M. Bökstedt, I. Madsen, Algebraic K-theory of local number fields: the unramified case, in Prospects in Topology (Princeton, 1994), Ann. of Math. Stud., vol. 139, Princeton Univ. Press, Princeton, NJ, 1995, pp. 28-57. MR 97e:19004

[Br] L. Breen, Extensions du groupe additif, Publ. Math. I.H.E.S. 48 (1978) 39-123. MR 81f:14011

[Bro] W. Browder, Torsion in $H$-spaces, Annals of Math. 74 (1961) 24-51. MR 23:A2201

[Brun] M. Brun, Spectral sequences for topological Hochschild homology, Thesis, Aarhus University, 1997.

[Brun1] M. Brun, Topological Hochschild homology of $Z / p^{n}$, J. Pure Appl. Algebra (to appear).

[BMMS] R. R. Bruner, J. P. May, J. E. McClure, M. Steinberger, $H_{\infty}$-ring spectra and their applications, Lecture Notes in Mathematics 1176, Springer-Verlag, 1980. MR 88e:55001

[BAG] Buenos Aires Cyclic Homology Group, Cyclic homology of algebras with one generator, K-Theory 5 (1991) 51-68. MR 93b:19002

[CLM] F. R. Cohen, T. J. Lada, J. P. May, The Homology of Iterated Loop Spaces, Springer LNM 533, 1976. MR 55:9096

[DM] B. Dundas, R. McCarthy, Stable K-theory and topological Hochschild homology, Annals of Math. 140 (1994) 685-689. MR 96e:19005a,b

[DM1] B. Dundas, R. McCarthy, Topological Hochschild homology of ring functors and exact categories, J. Pure Appl. Algebra 109 (1996) 231-294. MR 97i:19001

[EKMM] A. D. Elmendorf, I. Kriz, M. A. Mandell, J. P. May, Rings, Modules and Algebras in Stable Homotopy Theory, AMS Mathematical Surveys and Monographs vol. 47, 1997. MR 97a:55006

[FP] V. Franjou, T. Pirashvili, On Mac Lane cohomology for the ring of integers, Topology 37 (1998) 109-114. MR 98h:19002

[HM] L. Hesselholt, I. Madsen, On the K-theory of finite algebras over Witt vectors of perfect fields, Topology 36 (1996) 29-101. MR 97i:19002

[JP] M. Jibladze, T. Pirashvili, Cohomology of algebraic theories, J. of Algebra 137 (1991) 253-296. MR 92f:18005

[K] C. Kassel, La K-theorie stable, Bull. Soc. Math. France 110 (1982) 381-416. MR 84f: 18018

[L] A. Lindenstrauss, Topological Hochschild homology of extensions of $\mathbf{Z} / p \mathbf{Z}$ by polynomials, and of $\mathbf{Z}[x] /\left(x^{n}\right)$ and $\mathbf{Z}[x] /\left(x^{n}-1\right), K$-Theory 10, 239-265, 1996. MR 98e:19001

[L1] A. Lindenstrauss, The topological Hochschild homology of the Gaussian integers, Amer. J. Math. 118 (1996) 1011-1036. MR 98b:19002

[L2] A. Lindenstrauss, A relative spectral sequence for topological Hochschild homology of spectra, J. Pure Appl. Algebra (to appear).

[Mac] S. Mac Lane, Categories for the working mathematician, Graduate Texts in Mathematics 5, Springer-Verlag, 1971. MR 50:7275

[M] I. Madsen, Algebraic K-theory and traces, in Current Developments in Mathematics, 191-321, International Press (1996). MR 98g:19004

[M1] I. Madsen, Higher torsion in $S G$ and BSG, Math. Z. 143 (1975) 55-80. MR 51:11503

[May] J. P. May, $E_{\infty}$ ring spaces and $E_{\infty}$ ring spectra, Lecture Notes in Mathematics 577, Springer-Verlag, 1977. MR 58:13008

[May1] J. P. May, Simplicial Objects in Algebraic Topology, D. Van Nostrand Company, Inc., 1967. MR 36:5942

[PW] T. Pirashvili, F. Waldhausen, Mac Lane homology and topological Hochschild homology, J. Pure Appl. Algebra 82 (1992) 81-98. MR 93k:16016.

[Q] D. Quillen, Homotopical Algebra, Lecture Notes in Mathematics 43, Springer-Verlag, 1967. MR 36:6480

[S] J-P. Serre, Local Fields, Graduate Texts in Mathematics 67, Springer-Verlag, 1979. MR 82e:12016

Department of Mathematics, Indiana University, Bloomington, Indiana 47405

E-mail address: ayelet@math.indiana.edu

Department of Mathematics, Aarhus University, DK-8000 Aarhus, Denmark

E-mail address: imadsen@imf.au.dk 